\title{
Lorentz Group in Ray and Polarization Optics
}

\author{
S. Başkal 1 \\ Department of Physics, Middle East Technical University, 06531 Ankara, \\ Turkey \\ Y. S. Kim 2 \\ Department of Physics, University of Maryland, \\ College Park, Maryland 20742, U.S.A.
}

\begin{abstract}
While the Lorentz group serves as the basic language for Einstein's special theory of relativity, it is turning out to be the basic mathematical instrument in optical sciences, particularly in ray optics and polarization optics. The beam transfer matrix, commonly called the $A B C D$ matrix, is shown to be a two-by-two representation of the Lorentz group applicable to the three-dimensional space-time consisting of two space and one time dimensions. The Jones matrix applicable to polarization states turns out to be the two-by-two representations of the Lorentz group applicable to the four-dimensional space-time consisting of three space and one time dimensions. The four-by-four Mueller matrix applicable to the Stokes parameters as well as the Poincaré sphere are both shown to be the representations of the Lorentz group.
\end{abstract}

To be included as Chapter 10 in "Mathematical Optics: Classical, Quantum and Imaging Methods" edited by Vasudevan Lakshminarayanan (Taylor and Francis, New York).

\footnotetext{
${ }^{1}$ electronic address: baskal@newton.physics.metu.edu.tr

${ }^{2}$ electronic address: yskim@physics.umd.edu
} 


\section{Introduction}

One complex number contains two independent parameters. One two-by-two complex matrix with its four elements contains eight independent parameters. The unit determinant condition reduces the number of independent parameters to six. These matrices form a group which is called the twodimensional special linear group and is denoted as $S L(2, c)$.

This group has two important subgroups. If we choose only Hermitian matrices, this group is $S U(2)$ which is the two-dimensional rotation group describing the rotations of electron spins. Its correspondence with the threedimensional rotation group is well known.

From the group $S L(2, c)$, we can choose matrices with real elements. As in the case of the $S U(2)$ subgroup, these two-by-two real matrices contain three independent parameters forming the $S L(2, r)$ or $S p(2)$ subgroups. In paraxial ray optics, reflections and refractions are governed by such matrices and are called the $A B C D$ matrices (Azzam et al. 1977, Saleh et al. 2007). Thus we shall collectively refer these real subgroups as the $A B C D$ group.

In this report, we first discuss physical applications of this three-parameter $A B C D$ group. It is noted that the $A B C D$ matrix can be decomposed into three easy-to-understand rotation and squeeze matrices, which can then be rotated into a form having equal diagonal elements and two independent parameters (Başkal et al. 2009, Başkal et al. 2010). We shall discuss how this process can be developed using optical instruments for periodic systems, such as laser cavities and multilayer optics.

While the group $S U(2)$ corresponds to $S O(3)$ consisting of three-by-three matrices, the $A B C D$ group corresponds to the set of three-by-three matrices of Lorentz transformations applicable to the three-dimensional space consisting of one time dimension and two space dimensions. Lorentz transformations applicable to $z$ and $x$ directions and rotations around the $y$ axis are governed by this group, which is called $S O(2,1)$.

Consequently, this aspect of the $A B C D$ group allows us to study the essential features of Wigner's little group (Wigner 1939) dictating the internal space-time symmetries of relativistic particles moving along the $z$ direction. If we add the rotational degree of freedom around the $z$ axis, the group can be extended to four-by-four matrices applicable four-dimensional Minkowskian space consisting of three space dimensions and one time dimension.

Using the two-by-two matrix corresponding to the rotation around the $z$ axis, we can extend the three-parameter $A B C D$ group to the six-parameter 
$S L(2, c)$ group and thereafter to the six parameter Lorentz group. In this way we can move into polarization optics.

The physics of light polarization has a long history (Saleh et al. 2007,Brosseau 1998). The basic mathematical instruments in this field are the two-bytwo matrix formalism for Jones vectors (Jones 1941, 1947) and the fourby-four Mueller (Soleillet 1929, Mueller 1943) matrix formalism for the four Stokes (Stokes 1852) parameters. For many years, it was known that the set of matrices applicable to the two-component Jones vector has the $S U(2)$ symmetry. It is shown in this note that this $S U(2)$ symmetry can be extended to that of $S L(2, c)$, if we take into account different attenuation rates for the two different polarization directions (Opatrny et al.1993, Han et al. 1997, Ben-Aryeh 2005).

In this report, we address the issue of unifying these two mathematical devices into one mathematical formalism. We show that the Jones vector and the Stokes parameters are two-by-two and four-by-four representations of the same Lorentz group. Then the question is why we need the Stokes parameters with a more complicated four-by-four matrices. The four-byfour formalism can tell the degree of coherence between the two orthogonal polarization components. However, the Lorentz symmetry cannot change the decoherence parameter, in as much as the same way as this symmetry cannot change the mass of a given particle. In order to address this issue, we use the Poincaré sphere.

The Poincare sphere is a very useful and elegant graphical method to represent the polarization state of light (Poincaré 1982) In the past it was regarded as a geometry of the three-dimensional sphere with a fixed radius (Born et al. 1980). This sphere cannot accommodate the symmetry of the Lorentz group. Recently, it was noted by the present authors that this three-dimensional geometry can be extended to the four-dimensional Lorentz group (Başkal et al. 2006). It was noted also that this extended Poincaré sphere, with its Lorentz symmetry, cannot accommodate the variation of the decoherence parameter. However, it is possible to introduce two coupled Poincaré spheres using the $S O(3,2)$ deSitter group (Başkal et al. 2006). This enlarged symmetry allows variations of the decoherence parameter.

The loss of coherence leads to an increase in the entropy of the system. Indeed, the entropy can be computed from this extended Poincaré sphere. By coupling two Poincaré spheres, we can transfer the entropy from one sphere to the other. This could serve as another example of Feynman's rest of the universe (Feynman 1972). We note here that two coupled harmonic 
oscillators could serve as an illustrative example of Feynman's rest of the universe (Han et al. 1999).

In Sec. 2, we start with the six generators of the Lorentz group and their closed set of commutation relations. Starting from the two-by-two Pauli matrices, there are six linearly independent two-by-two matrices. It is noted that the three of these matrices are real, and the other three are purely imaginary. The $A B C D$ matrix is generated by those imaginary matrices.

In Sec. 3, it is shown that the optical $A B C D$ matrix can be decomposed into a product of three convenient matrices which are rotation and squeeze matrices. In Sec. 4, we use these properties to deal with periodic systems in optics.

In Sec. 5, the mathematical language of the $A B C D$ matrix is translated into that of the internal space-time symmetries of relativistic elementary particles. We note that the Wigner decomposition and the Bargmann decomposition can be explained in terms of the decomposition properties of the $A B C D$ matrix.

In Sec. 6, it is shown that the two-by-two Jones vector formalism is a representation of the Lorentz group. We add a squeeze matrix to the conventional $S U(2)$ formalism. The symmetry group is $S L(2, c)$ corresponding to the full Lorentz group with six independent parameters. From this, it is possible to construct a four-by-four representation of the Lorentz group. In Sec. 7, we show that the Mueller matrix applicable to the four Stokes parameters is the four-by-four representation of the Lorentz group.

Unlike the case of the two-by-two representation applicable to the Jones vectors, the four-by-four representation can deal with the decoherence problems. On the other hand, the decoherence parameter remains as a Lorentzinvariant parameter. In order to deal with this problem, we enlarge the symmetry group from the traditional Lorentz group of $S O(3,1)$ to the deSitter group of $O(3.2)$. We carry out this operation by extending the concept of the Poincaré sphere.

In Sec. 8, we first extend the traditional three-dimensional sphere to a four-dimensional object, in order to take into account all the symmetry contents of the Stokes parameters. We then introduce two Poincarśs spheres coupled to each other through the symmetry of the $O(3,2)$ deSitter group. This allows the exchange of the decoherence parameters between the two spheres. Furthermore, this picture of the two coupled Poincare spheres constitute another example of Feynman's rest of the universe (Feynman 1972). 


\section{Group of ABCD Matrices}

The Lorentz group is the group of four-by-four matrices applicable to the four-dimensional Minkowskian space of $(x, y, z, t)$. The group is generated by three rotation generators $J_{i}$ and three boost generators $K_{i}$, satisfying a closed set of commutation relations

$$
\left[J_{i}, J_{j}\right]=i \epsilon_{i j k} J_{k}, \quad\left[J_{i}, K_{j}\right]=i \epsilon_{i j k} K_{k}, \quad\left[K_{i}, K_{j}\right]=-i \epsilon_{i j k} J_{k} .
$$

These generators are all four-by-four matrices, and we shall give their explicit expressions in later sections.

Fortunately, there is a set of two-by-two matrices which satisfy the same commutation relations. We can write them as

$$
J_{1}=\frac{1}{2}\left(\begin{array}{ll}
0 & 1 \\
1 & 0
\end{array}\right), \quad J_{2}=\frac{1}{2}\left(\begin{array}{cc}
0 & -i \\
i & 0
\end{array}\right), \quad J_{3}=\frac{1}{2}\left(\begin{array}{cc}
1 & 0 \\
0 & -1
\end{array}\right) .
$$

They are the Pauli spin matrices which are very familiar to us. They are Hermitian matrices.

The boost generators $K_{i}$ can take the form of $i J_{i}$, or explicitly

$$
K_{1}=\frac{1}{2}\left(\begin{array}{cc}
0 & i \\
i & 0
\end{array}\right), \quad K_{2}=\frac{1}{2}\left(\begin{array}{cc}
0 & 1 \\
-1 & 0
\end{array}\right), \quad K_{3}=\frac{1}{2}\left(\begin{array}{cc}
i & 0 \\
0 & -i
\end{array}\right) .
$$

Unlike $J_{i}$, these matrices are anti-Hermitian.

The group of two-by-two matrices generated by the above six two-by-two matrices is called $S L(2, c)$. Since the generators of this group share the same set of commutation relations as the Lorentz group, they are said to be locally isomorphic to each other. In this paper, we shall avoid this mathematical word, and simply say that $S L(2, c)$ is "like" the Lorentz group.

There are a number of interesting subgroup of this $S L(2, c)$ group. The three generators $J_{i}$ generates the $S U(2)$ subgroup which is like the threedimensional rotation group. This aspect is well known. Three $K_{i}$ alone do not form a closed set of commutation relations.

Among the six generators, $J_{2}, K_{1}$, and $K_{3}$ are pure imaginary, and they generate two-by-two matrices with real elements. Furthermore they satisfy the closed set of commutation relations

$$
\left[J_{2}, K_{1}\right]=-i K_{3}, \quad\left[J_{2}, K_{3}\right]=i K_{1}, \quad\left[K_{1}, K_{3}\right]=i J_{2} .
$$

The group of two-by-two matrices generated by these three matrices is called $S p(2)$ or the two-dimensional symplectic group. It is like the Lorentz group 
applicable to the three-dimensional space of $(z, x, t)$. However, this group constitutes the fundamental mathematical language for the optical beam transfer matrix often called the $A B C D$ matrix.

The $A B C D$ matrix is a two-by-two matrix with real elements, and its determinant is one. There are therefore three independent parameters. These elements are determined by optical materials and how they are arranged. The purpose of this note is to explore its mathematical properties which can address more fundamental issues in physics.

First of all, the trace of this matrix could be less than two, equal to two, or greater than two. We are interested in what physical conclusions we can derive from these numbers.

In order to bring the $A B C D$ matrix to the form which will describe the particle symmetries, we should first transform it into the equi-diagonal form where the two diagonal elements are equal to each other (Başkal et al. 2009, Başkal et al. 2010). We can achieve this goal by a similarity transformation with a rotation matrix. Thus, this rotation angle becomes one of the three independent parameters, and the equi-diagonal $A B C D$ matrix has two independent parameters.

We shall call this equi-diagonal matrix the core of the $A B C D$ matrix, and use the notation $[A B C D]$. This matrix is not always diagonalized. This creates non-trivial problem. We shall examine how optical devices, especially periodic systems, can lead us to a better understanding of the problem. For this purpose, we discuss laser cavities and multilayer systems in detail.

If the trace is less than two, the core can be written as

$$
[A B C D]=\left(\begin{array}{cc}
\cos (\gamma / 2) & -e^{\eta} \sin (\gamma / 2) \\
e^{-\eta} \sin (\gamma / 2) & \cos (\gamma / 2)
\end{array}\right) .
$$

The diagonal elements are equal and smaller than one.

If the trace is greater than two, the core takes the form

$$
[A B C D]=\left(\begin{array}{cc}
\cosh (\gamma / 2) & e^{\eta} \sinh (\gamma / 2) \\
e^{-\eta} \sinh (\gamma / 2) & \cosh (\gamma / 2)
\end{array}\right) .
$$

Here again the diagonal elements are equal, but they are greater than one.

If the trace is equal to two, the $[A B C D]$ matrix becomes

$$
[A B C D]=\left(\begin{array}{cc}
1 & -\gamma \\
0 & 1
\end{array}\right) .
$$

This matrix also has the same diagonal element, and they are equal to one. 
The triangular matrix of Eq.(17) cannot be diagonalized. As for the matrices of Eq.(5) and Eq.(6), they can be diagonalized, but not by rotation alone. These mathematical subtleties are not well known. The purpose of this report is to show how much physics we can understand by studying this mathematical subtleties.

The mathematics of group theory allows us to write down a four-by-four Lorentz-transformation matrix for every two-by-two matrix discussed in this paper. In this way, the three matrices given in Eq.(5), Eq.(66), and Eq.(17) lead to the internal space-time symmetries of elementary particles. They respectively correspond to the symmetries of massive, imaginary-mass, and massless particles, respectively (Wigner 1939, Kim et al. 1986).

\section{Decomposition of the ABCD Matrix}

We are interested in writing the three different forms of the core matrix in one expression.

$$
[A B C D]=\exp \left\{\frac{1}{2}\left(\begin{array}{cc}
0 & -x-y \\
x-y & 0
\end{array}\right)\right\}
$$

where the parameters $x$ and $y$ are determined by the optical materials and how they are arranged. The exponent of this matrix is

$$
\frac{1}{2}\left(\begin{array}{cc}
0 & -x-y \\
x-y & 0
\end{array}\right) .
$$

If $x>y$, the exponent becomes

$$
\frac{\gamma}{2}\left(\begin{array}{cc}
0 & -\exp (\eta) \\
\exp (-\eta) & 0
\end{array}\right)
$$

which leads to the core matrix of Eq.(15) with

$$
\begin{aligned}
& \gamma=\sqrt{x^{2}-y^{2}} \\
& e^{\eta}=\sqrt{\frac{x+y}{x-y}} .
\end{aligned}
$$

The core matrix $[A B C D]$ can be written as a similarity transformation

$$
[A B C D]=B(\eta) R(\theta) B(-\eta)
$$


with

$$
\begin{aligned}
& B(\eta)=\left(\begin{array}{cc}
e^{\eta / 2} & 0 \\
0 & e^{-\eta / 2}
\end{array}\right), \\
& R(\theta)=\left(\begin{array}{cc}
\cos (\theta / 2) & -\sin (\theta / 2) \\
\sin (\theta / 2) & \cos (\theta / 2)
\end{array}\right),
\end{aligned}
$$

where $\gamma$ is now replaced by the rotation angle $\theta . R(\theta)$ is a rotation matrix, and $B(\eta)$ is a squeeze matrix.

If $x<y$, the exponent becomes

$$
\frac{\gamma}{2}\left(\begin{array}{cc}
0 & -\exp (\eta) \\
-\exp (-\eta) & 0
\end{array}\right)
$$

leading to the core matrix of Eq.(6)

$$
\begin{aligned}
& \gamma=\sqrt{y^{2}-x^{2}}, \\
& e^{\eta}=\sqrt{\frac{x+y}{y-x}} .
\end{aligned}
$$

The $[A B C D]$ matrix can now be decomposed into a similarity transformation

$$
[A B C D]=B(\eta) S(-\lambda) B(-\eta)
$$

with

$$
S(\lambda)=\left(\begin{array}{cc}
\cosh (\lambda / 2) & \sinh (\lambda / 2) \\
\sin (\lambda / 2) & \cosh (\lambda / 2)
\end{array}\right)
$$

where $\gamma$ is replaced by the boost parameter $\lambda$. The matrix $B(\eta)$ takes the diagonal form given in Eq.(12) with $\eta$ defined in Eq.(15). $S(\lambda)$ is a squeeze matrix.

If $x=y$, the exponent becomes

$$
\left(\begin{array}{cc}
1 & -x \\
0 & 1
\end{array}\right)
$$

with $x=y=\gamma$.

We now have combined three different expressions for the core of the $A B C D$ matrix into one exponential form of Eq.(8) . This form can be decomposed into three matrices constituting a similarity transformation. 
There is another form of decomposition known as the Bargmann decomposition (Bargmann 1947), which states that the core of the $A B C D$ matrix can be written as

$$
[A B C D]=R(\alpha) S(-2 \chi) R(\alpha),
$$

where the forms of the rotation matrix $R$ and the squeeze matrix $S$ are given as in Eq.(13) and Eq.(17) respectively. If we carry out the matrix multiplication, the $[A B C D]$ matrix becomes

$$
\left(\begin{array}{cc}
(\cosh \chi) \cos \alpha & -\sinh \chi-(\cosh \chi) \sin \alpha \\
-\sinh \chi+(\cosh \chi) \sin \alpha & (\cosh \chi) \cos \alpha
\end{array}\right) .
$$

This matrix also has two independent parameters $\alpha$ and $\chi$. We can write these parameters in terms of $\theta$ and $\eta$ by comparing the matrix elements. For instance, if $x>y$, the diagonal elements lead to

$$
\cos (\theta / 2)=(\cosh \chi) \cos \alpha
$$

The off-diagonal elements lead to

$$
e^{2 \eta}=\frac{(\cosh \chi) \sin \alpha+\sinh \chi}{(\cosh \chi) \sin \alpha-\sinh \chi} .
$$

As for physical applications, let us consider periodic systems, such as laser cavities and multilayer systems. The exponential form given in Eq.(8) tells us that it is a matter of replacing the $\theta$ parameter by $N \theta$ for $\mathrm{N}$ repeated applications (Başkal et al. 2010). Let us see some examples.

\section{Periodic Systems in Ray optics}

Let us summarize the content of Sec. 3. First of all, the optical $A B C D$ matrix can be brought to an equi-diagonal form by a similarity transformation, and we call this equi-diagonal matrix the core of $A B C D$, and use the notation $[A B C D]$. Thus,

$$
(A B C D)=T[A B C D] T^{-1}
$$

where we use $(A B C D)$ for the original $A B C D$ matrix. The transformation matrix can be a rotation or a triangular matrix. In addition, the core matrix can be written as a Wigner decomposition of the form

$$
[A B C D]=B(\eta) W(\gamma) B(-\eta)
$$


which is another similarity transformation with $B(\eta)$ given in Eq.(13). The $W(\gamma)$ is one of the three matrices

$$
\left(\begin{array}{cc}
\cos (\gamma / 2) & -\sin (\gamma / 2) \\
\sin (\gamma / 2) & \cosh (\gamma / 2)
\end{array}\right), \quad\left(\begin{array}{cc}
\cosh (\gamma / 2) & -\sinh (\gamma / 2) \\
-\sinh (\gamma / 2) & \cosh (\gamma / 2)
\end{array}\right), \quad\left(\begin{array}{cc}
1 & -\gamma \\
0 & 1
\end{array}\right)
$$

and

$$
(A B C D)=\left[\begin{array}{ll}
T B & B(\eta)
\end{array}\right] W(\gamma)[T B(\eta)]^{-1} .
$$

For repeated applications of the $A B C D$ matrix we need an expression of the form $(A B C D)^{N}$. Thanks to this form of similarity transformation, $(A B C D)^{N}$ is now simplified as

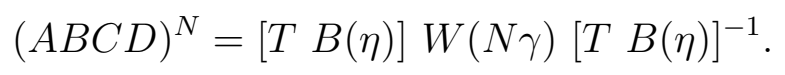

Thus the problem of periodic systems in optics is to find the core matrix $[A B C D]$.

\subsection{Laser Cavities}

As the first example, let us consider the laser cavity consisting of two identical concave mirrors separated by a distance $d$. Then the $A B C D$ matrix for a round trip of one beam is

$$
\left(\begin{array}{cc}
1 & 0 \\
-2 / R & 1
\end{array}\right)\left(\begin{array}{ll}
1 & d \\
0 & 1
\end{array}\right)\left(\begin{array}{cc}
1 & 0 \\
-2 / R & 1
\end{array}\right)\left(\begin{array}{ll}
1 & d \\
0 & 1
\end{array}\right)
$$

where the matrices

$$
\left(\begin{array}{cc}
1 & 0 \\
-2 / R & 1
\end{array}\right), \quad\left(\begin{array}{ll}
1 & d \\
0 & 1
\end{array}\right)
$$

are the mirror and translation matrices respectively. The parameters $R$ and $d$ are the radius of the mirror and the mirror separation respectively. This form is quite familiar to us from the laser literature (Yariv 1975, Haus 1984, Hawkes et al. 1995).

However, the main issue here is how to alleviate the problem of taking the Nth power of chains of matrices which corresponds to the repetition of this process for many times. This can be achieved when the matrices in Eq.(28) can be brought to an equi-diagonal form and eventually to a form 
of the Wigner decomposition. Thus, we are interested in finding the core of Eq.(28). For his purpose, we rewrite the matrix of Eq.(28) as

$$
\begin{aligned}
& \left(\begin{array}{cc}
1 & -d / 2 \\
0 & 1
\end{array}\right)\left(\begin{array}{cc}
1 & d / 2 \\
0 & 1
\end{array}\right)\left(\begin{array}{cc}
1 & 0 \\
-2 / R & 1
\end{array}\right)\left(\begin{array}{cc}
1 & d / 2 \\
0 & 1
\end{array}\right)^{2} \\
& \times\left(\begin{array}{cc}
1 & 0 \\
-2 / R & 1
\end{array}\right)\left(\begin{array}{cc}
1 & d / 2 \\
0 & 1
\end{array}\right)\left(\begin{array}{cc}
1 & d / 2 \\
0 & 1
\end{array}\right) .
\end{aligned}
$$

In this way, we translate the system by $-d / 2$ using a translation matrix given in Eq.(29), and write the $A B C D$ matrix of Eq.(28) as

$$
\left(\begin{array}{cc}
1 & -d / 2 \\
0 & 1
\end{array}\right)\left[\left(\begin{array}{cc}
1-d / R & d-d^{2} / 2 R \\
-2 / R & 1-d / R
\end{array}\right)\right]^{2}\left(\begin{array}{cc}
1 & d / 2 \\
0 & 1
\end{array}\right) .
$$

We are thus led to concentrate on the matrix in the middle

$$
\left(\begin{array}{cc}
1-d / R & d-d^{2} / 2 R \\
-2 / R & 1-d / R
\end{array}\right)
$$

which can be written as

$$
\left(\begin{array}{cc}
\sqrt{d} & 0 \\
0 & 1 / \sqrt{d}
\end{array}\right)\left(\begin{array}{cc}
1-d / R & 1-d / 2 R \\
-2 d / R & 1-d / R
\end{array}\right)\left(\begin{array}{cc}
1 / \sqrt{d} & 0 \\
0 & \sqrt{d}
\end{array}\right) .
$$

It is then possible to decompose the $A B C D$ matrix into

$$
E C^{2} E^{-1}
$$

with

$$
\begin{aligned}
C & =\left(\begin{array}{cc}
1-d / R & 1-d / 2 R \\
-2 d / R & 1-d / R
\end{array}\right), \\
E & =\left(\begin{array}{cc}
1 & -d / 2 \\
0 & 1
\end{array}\right)\left(\begin{array}{cc}
\sqrt{d} & 0 \\
0 & 1 / \sqrt{d}
\end{array}\right) .
\end{aligned}
$$

The $C$ matrix now contains only dimensionless numbers, and it can be written as

$$
C=\left(\begin{array}{cc}
\cos (\gamma / 2) & e^{\eta} \sin (\gamma / 2) \\
-e^{-\eta} \sin (\gamma / 2) & \cos (\gamma / 2)
\end{array}\right)
$$

with

$$
\begin{aligned}
& \cos (\gamma / 2)=1-\frac{d}{R}, \\
& e^{\eta}=\sqrt{\frac{2 R-d}{4 d}}
\end{aligned}
$$


Here both $d$ and $R$ are positive, and the restriction on them is that $d$ be smaller than $2 R$. This is the stability condition frequently mentioned in the literature (Haus 1984, Hawkes et al. 1995).

Thus, the $[A B C D]$ core matrix is $C^{2}$, and takes the form

$$
[A B C D]=\left(\begin{array}{cc}
\cos (\gamma) & e^{\eta} \sin (\gamma) \\
-e^{-\eta} \sin (\gamma) & \cos (\gamma)
\end{array}\right)
$$

and the similarity transformation which connects this core matrix with the original $A B C D$ matrix of Eq.(28) is $E$ given in Eq.(35).

\subsection{Multilayer Optics}

We consider an optical beam going through a periodic medium with two different refractive indices. If the beam traveling in the first medium hits the second medium, it is partially transmitted and partially reflected. In order to maintain the continuity of the Poynting vector, we define the electric fields as

$$
\begin{aligned}
& E_{1}^{( \pm)}=\frac{1}{\sqrt{n_{1}}} \exp \left( \pm i k_{1} z-\omega t\right) \\
& E_{2}^{( \pm)}=\frac{1}{\sqrt{n_{2}}} \exp \left( \pm i k_{2} z-\omega t\right)
\end{aligned}
$$

for the optical beams in the first and second media respectively. The superscript $(+)$ and $(-)$ are for the incoming and reflected rays respectively.

These two optical rays are related by the two-by-two $A B C D$ matrix, according to

$$
\left(\begin{array}{l}
E_{2}^{(+)} \\
E_{2}^{(-)}
\end{array}\right)=\left(\begin{array}{ll}
A & B \\
C & D
\end{array}\right)\left(\begin{array}{l}
E_{1}^{(+)} \\
E_{1}^{(-)}
\end{array}\right) .
$$

Of course the elements of this matrix are determined by transmission coefficients as well as the phase shifts the beams experience while going through the media (Azzam et al. 1977, Georgieva et al. 2001).

When the beam goes through the first medium to the second, we may use the the boundary matrix given in (Azzam et al. 1977) and in (Monzon et al. 2000, Monzon et al.2002). In terms of the refractive indexes $n_{1}$ and $n_{2}$, this matrix is

$$
Q(\sigma)=\left(\begin{array}{cc}
\cosh (\sigma / 2) & \sinh (\sigma / 2) \\
\sinh (\sigma / 2) & \cosh (\sigma / 2)
\end{array}\right)
$$


where one can write the $\sigma$ parameter as

$$
\cosh \left(\frac{\sigma}{2}\right)=\frac{n_{1}+n_{2}}{2 \sqrt{n_{1} n_{2}}}, \quad \sinh \left(\frac{\sigma}{2}\right)=\frac{n_{1}-n_{2}}{2 \sqrt{n_{1} n_{2}}}
$$

The boundary matrix for the beam going from the second medium should be $Q(-\sigma)$.

In addition, we have to consider the phase shifts the beams have to go through. When the beam goes trough the first media, we can use the phaseshift matrix

$$
P\left(\delta_{1}\right)=\left(\begin{array}{cc}
e^{-i \delta_{1} / 2} & 0 \\
0 & e^{i \delta_{1} / 2}
\end{array}\right)
$$

and a similar expression for $P\left(\delta_{2}\right)$ for the second medium. The phase shift $\delta$ is determined by the wave number and the thickness of the medium.

We are thus led to consider one complete cycle starting from the midpoint of the second medium, and write

$$
P\left(\delta_{2} / 2\right) Q(\sigma) P\left(\delta_{1}\right) Q(-\sigma) P\left(\delta_{2} / 2\right) .
$$

There are two questions in regards to the above matrix multiplication. One is whether each matrix in this expression can be converted into a matrix with real elements and the other is whether the result of this matrix multiplication accommodates Wigner and Bargmann decompositions. In order to answer the first question, let us consider the similarity transformation

$$
C_{1} P(\delta) Q(\sigma) C_{1}^{-1}
$$

with

$$
C_{1}=\frac{1}{\sqrt{2}}\left(\begin{array}{ll}
1 & i \\
i & 1
\end{array}\right)
$$

This transformation leads to

$$
R(\delta) Q(\sigma)
$$

where

$$
R(\delta)=\left(\begin{array}{cc}
\cos (\delta / 2) & -\sin (\delta / 2) \\
\sin (\delta / 2) & \cos (\delta / 2)
\end{array}\right)
$$

This notation is consistent with the rotation matrices used in Sec. 3 ,

Let us make another similarity transformation with

$$
C_{2}=\frac{1}{\sqrt{2}}\left(\begin{array}{cc}
1 & 1 \\
-1 & 1
\end{array}\right)
$$


This changes $Q(\sigma)$ into $B(\sigma)$ without changing $R(\delta)$, where

$$
B(\sigma)=\left(\begin{array}{cc}
e^{\sigma / 2} & 0 \\
0 & e^{-\sigma / 2}
\end{array}\right)
$$

again consistent with the $B(\eta)$ matrix used in Sec. 3 .

Thus the net similarity transformation matrix is (Georgieva et al. 2001)

$$
C=C_{2} C_{1}=\frac{1}{\sqrt{2}}\left(\begin{array}{cc}
e^{i \pi / 4} & e^{i \pi / 4} \\
-e^{-i \pi / 4} & e^{-i \pi / 4}
\end{array}\right)
$$

If we apply this similarity transformation to the long matrix chain of Eq.(44), it becomes another chain

$$
M=R\left(\delta_{2} / 2\right) B(\sigma) R\left(\delta_{1}\right) B(-\sigma) R\left(\delta_{2} / 2\right)
$$

where all the matrices are real.

Let us now address the main question of whether this matrix chain can be brought to one equi-diagonal matrix. We note first that the three middle matrices can be written in a familiar form

$$
\begin{aligned}
M & =B(\sigma) R\left(\delta_{1}\right) B(-\sigma) \\
& =\left(\begin{array}{cc}
\cos \left(\delta_{1} / 2\right) & -e^{\sigma} \sin \left(\delta_{1} / 2\right) \\
e^{-\sigma} \sin \left(\delta_{1} / 2\right) & \cos \left(\delta_{1} / 2\right)
\end{array}\right)
\end{aligned}
$$

However, due to the rotation matrix $R\left(\delta_{2} / 2\right)$ at the beginning and at the end of Eq.(52), it is not clear whether the entire chain can be written as a similarity transformation.

In order to resolve this issue, let us write Eq.(153) as a Bargmann decomposition

$$
R(\alpha) S(-2 \chi) R(\alpha)
$$

with its explicit expression given in Eq.(20). The parameters $\alpha$ and $\chi$ are related to $\sigma$ and $\delta_{1}$ by

$$
\begin{aligned}
& \cos \left(\delta_{1} / 2\right)=(\cosh \chi) \cos \alpha, \\
& e^{2 \sigma}=\frac{(\cosh \chi) \sin \alpha+\sinh \chi}{(\cosh \chi) \sin \alpha-\sinh \chi} .
\end{aligned}
$$


It is now clear that the entire chain of Eq.(44) can be written as another Bargmann decomposition

$$
M=R\left(\alpha+\delta_{2} / 2\right) S(-2 \chi) R\left(\alpha+\delta_{2} / 2\right) .
$$

Finally, this expression can be converted to a Wigner decomposition (Georgieva et al. 2003)

$$
M=B(\eta) R(\theta) B(-\eta)
$$

with

$$
\begin{aligned}
& \cos (\theta / 2)=(\cosh \chi) \cos \left(\alpha+\delta_{2} / 2\right), \\
& e^{2 \eta}=\frac{(\cosh \chi) \sin \left(\alpha+\delta_{2} / 2\right)+\sinh \chi}{(\cosh \chi) \sin \left(\alpha+\delta_{2} / 2\right)-\sinh \chi} .
\end{aligned}
$$

The decomposition of Eq.(157) allows us to deal with the periodic system of multilayers. For repeated application of $M$, we can now write

$$
M^{N}=B(\eta) R(N \theta) B(-\eta)
$$

\section{Space-time Symmetries}

In Sec. 3, we have seen that the two-by-two matrices can provide a very powerful language for optical systems. However, this language is not restricted to the two-dimensional world. It can be translated into the four-dimensional world of Einstein's special relativity where Lorentz transformations play the central role.

In mathematics, the group of two-by-two unimodular matrices is called $S L(2, c)$. The group of four-dimensional matrices performing Lorentz transformations on the Minkowskian four-vector $(t, z, x, y)$ is called the $S O(3,1)$ Lorentz group. The group $S L(2, c)$ has six generators, so does the $S O(3,1)$, but the corresponding matrices are two-by-two and four-by-four, respectively. Their generators satisfy the same set of commutation relations as in Eq.(1). This correspondence is called the local isomorphism between the $S L(2, c)$ and $S O(3,1)$ groups. The four-dimensional generators are

$$
J_{1}=\left(\begin{array}{cccc}
0 & 0 & 0 & 0 \\
0 & 0 & 0 & i \\
0 & 0 & 0 & 0 \\
0 & -i & 0 & 0
\end{array}\right), \quad J_{2}=\left(\begin{array}{cccc}
0 & 0 & 0 & 0 \\
0 & 0 & -i & 0 \\
0 & i & 0 & 0 \\
0 & 0 & 0 & 0
\end{array}\right), \quad J_{3}=\left(\begin{array}{cccc}
0 & 0 & 0 & 0 \\
0 & 0 & 0 & 0 \\
0 & 0 & 0 & -i \\
0 & 0 & i & 0
\end{array}\right)
$$


and

$$
K_{1}=\left(\begin{array}{cccc}
0 & 0 & i & 0 \\
0 & 0 & 0 & 0 \\
i & 0 & 0 & 0 \\
0 & 0 & 0 & 0
\end{array}\right), \quad K_{2}=\left(\begin{array}{cccc}
0 & 0 & 0 & i \\
0 & 0 & 0 & 0 \\
i & 0 & 0 & 0 \\
0 & 0 & 0 & 0
\end{array}\right), \quad K_{3}=\left(\begin{array}{cccc}
0 & i & 0 & 0 \\
i & 0 & 0 & 0 \\
0 & 0 & 0 & 0 \\
0 & 0 & 0 & 0
\end{array}\right) \text {. }
$$

This mathematical property allows us to explain events in Einstein's Lorentz-covariant world in terms of what we observe in optics laboratories.

\subsection{Two-by-two and four-by-four representations of the Lorentz group}

The content of this correspondence is somewhat complicated, but for the present purpose, we can start with the Minkowskian four-vector $(t, z, x, y)$ written as

$$
X=\left(\begin{array}{cc}
t+z & x-i y \\
x+i y & t-z
\end{array}\right)
$$

whose determinant

$$
t^{2}-z^{2}-x^{2}-y^{2}
$$

is left invariant under Lorentz transformations. Now consider a transformation

$$
X^{\prime}=G X G^{\dagger}
$$

where $G$ a unimodular matrix whose determinant is one. Let us write this matrix as

$$
G=\left(\begin{array}{ll}
\alpha & \beta \\
\gamma & \delta
\end{array}\right)
$$

where the elements can be complex numbers. If the determinant of this matrix is one, there are only six independent parameters. Thus this matrix can be generated by the six generators given in Eq.(2) and Eq.(3) .

The transformation of Eq. (64) can be explicitly written as

$$
\left(\begin{array}{cc}
t^{\prime}+z^{\prime} & x^{\prime}-i y^{\prime} \\
x^{\prime}+i y^{\prime} & t^{\prime}-z^{\prime}
\end{array}\right)=\left(\begin{array}{cc}
\alpha & \beta \\
\gamma & \delta
\end{array}\right)\left(\begin{array}{cc}
t+z & x-i y \\
x+i y & t-z
\end{array}\right)\left(\begin{array}{cc}
\alpha^{*} & \gamma^{*} \\
\beta^{*} & \delta^{*}
\end{array}\right)
$$

We can now translate this formula into

$$
\left(\begin{array}{c}
t^{\prime}+z^{\prime} \\
x^{\prime}-i y^{\prime} \\
x^{\prime}+i y^{\prime} \\
t^{\prime}-z^{\prime}
\end{array}\right)=\left(\begin{array}{llll}
\alpha \alpha^{*} & \alpha \beta^{*} & \beta \alpha^{*} & \beta \beta^{*} \\
\alpha \gamma^{*} & \alpha \delta^{*} & \beta \gamma^{*} & \beta \delta^{*} \\
\gamma \alpha^{*} & \gamma \beta^{*} & \delta \alpha^{*} & \delta \beta^{*} \\
\gamma \gamma^{*} & \gamma \delta^{*} & \delta \gamma^{*} & \delta \delta^{*}
\end{array}\right)\left(\begin{array}{c}
t+z \\
x-i y \\
x+i y \\
t-z
\end{array}\right)
$$


It can be seen that the above transformation matrix can be expressed in the form of a Kronecker product as

$$
G \otimes G^{*}
$$

where

$$
G^{*}=\left(\begin{array}{ll}
\alpha^{*} & \beta^{*} \\
\gamma^{*} & \delta^{*}
\end{array}\right)
$$

Then the components of $X^{\prime}$ are related to $\left(t^{\prime}, z^{\prime}, x^{\prime}, y^{\prime}\right)$ as

$$
\left(\begin{array}{l}
t^{\prime} \\
z^{\prime} \\
x^{\prime} \\
y^{\prime}
\end{array}\right)=\frac{1}{2}\left(\begin{array}{cccc}
1 & 1 & 0 & 0 \\
1 & -1 & 0 & 0 \\
0 & 0 & 1 & 1 \\
0 & 0 & i & -i
\end{array}\right)\left(\begin{array}{c}
t^{\prime}+z^{\prime} \\
t^{\prime}-z^{\prime} \\
x^{\prime}-i y^{\prime} \\
x^{\prime}+i y^{\prime}
\end{array}\right)
$$

Likewise, the two-by-two matrix for the four-momentum of the particle takes the form

$$
P=\left(\begin{array}{cc}
p_{0}+p_{z} & p_{x}-i p_{y} \\
p_{x}+i p_{y} & p_{0}-p_{z}
\end{array}\right)
$$

with $p_{0}=\sqrt{m^{2}+p_{z}^{2}+p_{x}^{2}+p_{z}^{2}}$. The transformation of this matrix takes the same form as that of the space-time four-vector given in Eq.(64) and Eq.(66).

\subsection{Internal Space-time Symmetries of Elementary Par- ticles}

These properties are applicable to many other branches of physics. For instance, one of the persisting problem is the internal space-time symmetry of elementary particles in Einstein's Lorentz-covariant world. The mathematics of group theory allows us to translate the rotation and squeeze matrices of Eq.(13) and Eq.(17) into the following four-by-four matrices respectively.

$$
\begin{aligned}
R(\theta) & =\left(\begin{array}{cccc}
1 & 0 & 0 & 0 \\
0 & \cos \theta & -\sin \theta & 0 \\
0 & \sin \theta & \cos \theta & 0 \\
0 & 0 & 0 & 1
\end{array}\right), \quad S(\lambda)=\left(\begin{array}{cccc}
\cosh \lambda & 0 & \sinh \lambda & 0 \\
0 & 1 & 0 & 0 \\
\sinh \lambda & 0 & \cosh \lambda & 0 \\
0 & 0 & 0 & 1
\end{array}\right) \\
B(\eta) & =\left(\begin{array}{cccc}
\cosh \eta & \sinh \eta & 0 & 0 \\
\sinh \eta & \cosh \eta & 0 & 0 \\
0 & 0 & 1 & 0 \\
0 & 0 & 0 & 1
\end{array}\right) .
\end{aligned}
$$


They are applicable to the Minkowskian four-vector $(x, y, z, t)$. The $R(\theta)$ matrix performs a rotation around the $y$ axis, and $S(\lambda)$ is for Lorentz boosts along the $x$ axis. The $B(\eta)$ matrix boosts the system along the $z$ direction.

Together with a rotation matrix around $z$ axis (Han et al. 1986)

$$
Z(\phi)=\left(\begin{array}{cccc}
1 & 0 & 0 & 0 \\
0 & 1 & 0 & 0 \\
0 & 0 & \cos \phi & -\sin \phi \\
0 & 0 & \sin \phi & \cos \phi
\end{array}\right),
$$

they constitute Wigner's little groups dictating internal space-time symmetries of massive and imaginary-mass particles (Wigner 1939). The triangular matrix of Eq.(7) leads to the little group for massless particles. The little groups are the subgroups of the Lorentz group whose transformations leave the four-momentum of a relativistic particle invariant.

It is possible to compute the two-by-two equivalent of the above $Z(\phi)$ matrix using the relation given in Eq.(64). It takes the form

$$
Z(\phi)=\left(\begin{array}{cc}
e^{i \phi / 2} & 0 \\
0 & e^{-i \phi / 2}
\end{array}\right)
$$

This matrix contains complex elements. This is the reason why it is not mentioned in our discussions of the $A B C D$ matrix. This rotation matrix will play an important role in polarization optics which will be discussed in Sec. 6 and Sec. 7 .

Let us go back to Eq.(15) which, according to Eq.(12), can be decomposed to a similarity transformation

$$
W(\eta, \theta)=B(\eta) R(\theta) B(-\eta) .
$$

We can write this decomposition with the four-by-four matrices given in Eq.(72).

Let us then consider a massive particle moving along the $z$ direction with the velocity parameter $v / c=\tanh \eta$, and its four-momentum

$$
(m \cosh \eta, m \sinh \eta, 0,0)
$$

where $m$ is the mass of the particle.

We can boost this particle using the boost matrix $B(-\eta)$, which is the inverse of the four-by-four matrix given in Eq.(72). The particle becomes at rest, with its four-momentum

$$
(m, 0,0,0)
$$




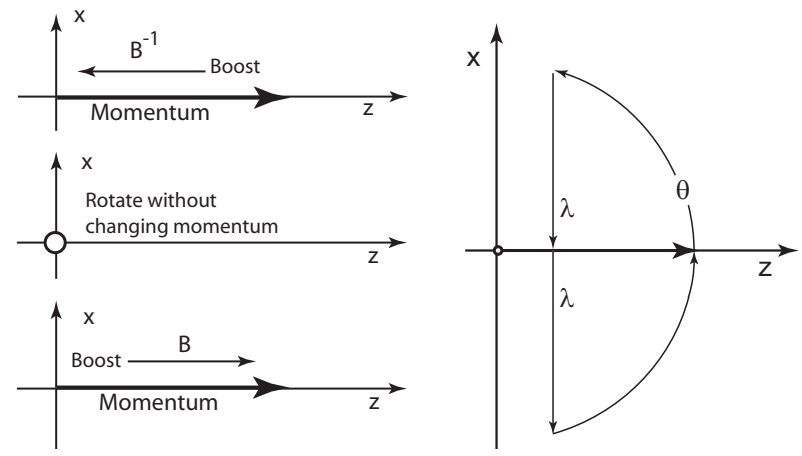

Figure 1: Wigner decomposition (left) and Bargmann decomposition (right). These figures illustrate momentum preserving transformations. In the Wigner transformation, a massive particle is brought to its rest frame. It can be rotated while the momentum remains the same. This particle is then boosted back to the frame with gaining original momentum. In the Bargmann decomposition, the momentum is rotated, boosted, and rotated to its original state.

and with zero velocity. The rotation matrix $R(\theta)$ rotates this particle without changing its momentum. During this process, the particle changes the direction of its spin. Finally, $B(\eta)$ boosts the particle and restores its momentum. In this way, the four-by-four expression for Eq.(12) changes the internal space-time structure of the particle.

One key question from this table is what happens to the $O(3)$-like little group when the particle momentum becomes infinity or its mass becomes zero. The question is whether the little group for a massive particles becomes that of a massless particle. The answer to this question is Yes, but this issue had a stormy history before this definitive answer (Kim et al. 1990). Indeed, when $\eta$ becomes infinity, the four-by-four form of $\mathrm{Eq}(\mathbf{7 5})$ becomes

$$
\left(\begin{array}{cccc}
1+\gamma^{2} / 2 & \gamma^{2} / 2 & \gamma & 0 \\
\gamma^{2} / 2 & 1+\gamma^{2} / 2 & \gamma & 0 \\
\gamma & \gamma & 1 & 0 \\
\gamma & \gamma & 0 & 1
\end{array}\right)
$$

When applied to the momentum of a massless particle moving in the negative $z$ direction with

$$
(p,-p, 0,0)
$$


it leaves the above four-momentum invariant, but on the other hand performs a gauge transformation when applied to the four-potentials of the electromagnetic field (Kim et al. 1990).

\section{Jones Vectors}

In studying polarized light propagating along the $z$ direction, the traditional approach is to consider the $x$ and $y$ components of the electric fields. Their amplitude ratio and the phase difference determine the state of polarization. Thus, we can change the polarization either by adjusting the amplitudes, by changing the relative phases, or both. For convenience, we call the optical device which changes amplitudes an "attenuator" and the device which changes the relative phase a "phase shifter."

The traditional language for this two-component light is the Jones-matrix formalism which is discussed in standard optics textbooks (Hecht 1970). In this formalism, the above two components are combined into one column matrix with the exponential form for the sinusoidal function

$$
\left(\begin{array}{l}
E_{x} \\
E_{y}
\end{array}\right)=\left(\begin{array}{l}
A \exp \left\{i\left(k z-\omega t+\phi_{1}\right)\right\} \\
B \exp \left\{i\left(k z-\omega t+\phi_{2}\right)\right\}
\end{array}\right)
$$

This column matrix is called the Jones vector (Jones 1941) .

The Jones-matrix formalism starts with the projection operator (Hecht 1970)

$$
\left(\begin{array}{ll}
1 & 0 \\
0 & 0
\end{array}\right)
$$

applicable to the Jones vector of Eq.(80). This operator keeps the $x$ component and completely eliminates the $y$-component of the electric field.

This is an oversimplification of the real world where the attenuation factor in the $y$ direction is greater than that of the $x$ direction. We shall replace this projection operator by an attenuation matrix which is closer to the real world.

In this note, we replace the projection operator of Eq.(81) by a squeeze matrix. There are two transverse directions which are perpendicular to each other. The absorption coefficient in one transverse direction could be different from the coefficient along the other direction. Thus, there is the "polarization" coordinate in which the absorption can be described by (Opatrny et 
al. 1993, Han et al. 1997, Ben-Aryeh 2005)

$$
\left(\begin{array}{cc}
e^{-\mu_{1}} & 0 \\
0 & e^{-\mu_{2}}
\end{array}\right)=e^{-\left(\mu_{1}+\mu_{2}\right) / 2}\left(\begin{array}{cc}
e^{\mu / 2} & 0 \\
0 & e^{-\mu / 2}
\end{array}\right)
$$

with $\mu=\mu_{2}-\mu_{1}$. Let us look at the projection operator of Eq.(81). Physically, it means that the absorption coefficient along the $y$ direction is much larger than that of the $x$ direction. The absorption matrix in Eq.(82) becomes the projection matrix if $\mu_{1}$ is very close to zero and $\mu_{2}$ becomes infinitely large. The projection operator of Eq.(81) is therefore a special case of the above attenuation matrix.

The attenuation matrix of Eq.(82) tells us that the electric fields are attenuated at two different rates. The exponential factor $e^{-\left(\mu_{1}+\mu_{2}\right) / 2}$ reduces both components at the same rate and does not affect the state of polarization. The effect of polarization is solely determined by the squeeze matrix

$$
B(\mu)=\left(\begin{array}{cc}
e^{\mu / 2} & 0 \\
0 & e^{-\mu / 2}
\end{array}\right)
$$

which is given in Eq.(13). This type of mathematical operation is quite familiar from studies of squeezed states of light, if not from Lorentz boosts of spinors. Thus, we are expanding the Jones-matrix formalism by replacing the projection operator of Eq.(81) by the squeeze operator in Eq.(83).

Another basic element is the optical filter with two different values of the index of refraction along the two orthogonal directions. The effect on this filter can be written as

$$
\left(\begin{array}{cc}
e^{i \delta_{1}} & 0 \\
0 & e^{i \delta_{2}}
\end{array}\right)=e^{i\left(\delta_{1}+\delta_{2}\right) / 2}\left(\begin{array}{cc}
e^{-i \delta / 2} & 0 \\
0 & e^{i \delta / 2}
\end{array}\right)
$$

with $\delta=\delta_{1}-\delta_{2}$. In measurement processes, the overall phase factor $e^{i\left(\delta_{1}+\delta_{2}\right) / 2}$ cannot be detected, and can therefore be deleted. The polarization effect of the filter is solely determined by the matrix

$$
Z(\delta)=\left(\begin{array}{cc}
e^{i \delta / 2} & 0 \\
0 & e^{-i \delta / 2}
\end{array}\right)
$$

which leads to a phase difference of $\delta$ between the $x$ and $y$ components. The mathematical expression for this matrix is given in Eq.(74). It has a different physical meaning in the symmetry of the Lorentz group. 
The polarization axes are not always the $x$ and $y$ axes. For this reason, we need the rotation matrix

$$
R(\theta)=\left(\begin{array}{cc}
\cos (\theta / 2) & -\sin (\theta / 2) \\
\sin (\theta / 2) & \cos (\theta / 2)
\end{array}\right)
$$

The traditional Jones-matrix formalism consists of systematic combinations of the above three components given in Eq.(81), Eq.(85) and Eq.(86).

\subsection{Squeeze and Phase shift}

The effect of the phase shift matrix $Z(\delta)$ of Eq. (85) on the Jones vector is well known, but the effect of the squeeze matrix of Eq.(83) is not addressed adequately in the literature. Let us discuss the combined effect of these two matrices. First of all both are diagonal and they commute with each other.

The effect of the squeeze matrix on the Jones vector is straight-forward and the net result is

$$
\left(\begin{array}{cc}
e^{\mu / 2} & 0 \\
0 & e^{-\mu / 2}
\end{array}\right)\left(\begin{array}{l}
E_{x} \\
E_{y}
\end{array}\right)=\left(\begin{array}{c}
e^{\mu / 2} E_{x} \\
e^{-\mu / 2} E_{y}
\end{array}\right) .
$$

This squeeze transformation expands one amplitude, while contracting the other so that the product of the amplitudes remain invariant. This squeeze transformation is illustrated in Fig. 2.

In order to illustrate phase shifts, we start with the Jones vector of the form

$$
\left(\begin{array}{c}
\exp (i k z) \\
\exp [i(k z-\pi / 2)]
\end{array}\right)
$$

whose real part is

$$
\left(\begin{array}{l}
x \\
y
\end{array}\right)=\left(\begin{array}{c}
\cos (k z) \\
\sin (k z)
\end{array}\right)
$$

which corresponds to a circular polarization with

$$
x^{2}+y^{2}=1 \text {. }
$$

If we apply the phase shift matrix, the resulting vector is

$$
\left(\begin{array}{l}
x \\
y
\end{array}\right)=\left(\begin{array}{c}
\cos (k z+\delta / 2) \\
\sin (k z-\delta / 2)
\end{array}\right)
$$




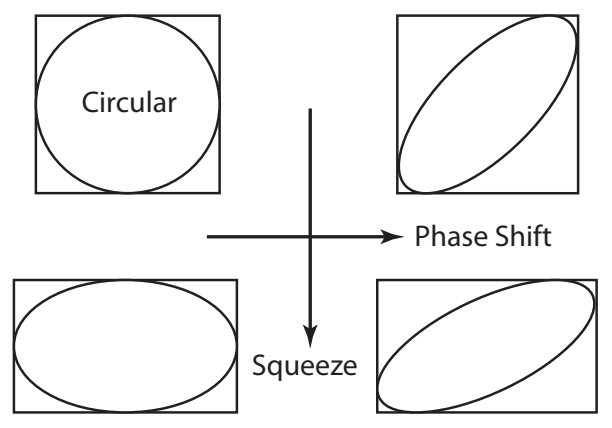

Figure 2: Squeeze and Phase Shift. Both squeeze and phase shifts result in elliptic deformations, but they are done differently.

which can be written as

$$
\left(\begin{array}{l}
x \\
y
\end{array}\right)=\left(\begin{array}{c}
\cos (k z-\pi / 4+\alpha) \\
\cos (k z-\pi / 4-\alpha)
\end{array}\right)
$$

with

$$
\alpha=\frac{\delta}{2}+\frac{\pi}{4}
$$

Then

$$
\begin{aligned}
& x+y=2(\cos \alpha) \cos (k z-\pi / 4), \\
& x-y=-2(\sin \alpha) \sin (k z-\pi / 4),
\end{aligned}
$$

and

$$
\frac{(x+y)^{2}}{4(\cos \alpha)^{2}}+\frac{(x-y)^{2}}{4(\sin \alpha)^{2}}=1 .
$$

This is an elliptic polarization.

The squeeze operation of Eq. (83) is relatively simple. It changes the amplitudes, and it commutes with the phase shift matrix. Thus, the combined effect could be illustrated in Fig. 2.

\subsection{Rotation of the Polarization Axes}

If the polarization coordinate is the same as the $x y$ coordinate where the electric field components take the form of Eq.(80), the attenuator is directly 
applicable to the Jones vector as in Eq. (87). If the polarization coordinate is rotated by an angle of $(\theta / 2)$, or by the matrix

$$
R(\theta)=\left(\begin{array}{cc}
\cos (\theta / 2) & -\sin (\theta / 2) \\
\sin (\theta / 2) & \cos (\theta / 2)
\end{array}\right),
$$

then the polarization coordinate makes an angle $(\theta / 2)$ with the $x y$ coordinate system. So the phase shifter takes the form

$$
\begin{aligned}
& Z(\theta, \delta)=R(\theta) P(\delta) R(-\theta) \\
& =\left(\begin{array}{cc}
\cos (\delta / 2)+i \sin (\delta / 2) \cos \theta & i \sin (\delta / 2) \sin \theta \\
i \sin (\delta / 2) \sin \theta & \cos (\delta / 2)-i \sin (\delta / 2) \cos \theta
\end{array}\right) .
\end{aligned}
$$

If the polarization coordinate system is rotated by $45^{\circ}$, the phase shifter matrix becomes

$$
Q(\delta)=\left(\begin{array}{cc}
\cos (\delta / 2) & i \sin (\delta / 2) \\
i \sin (\delta / 2) & \cos (\delta / 2)
\end{array}\right)
$$

In order to illustrate what this matrix does to the polarized beams, let us start with the circularly polarized wave

$$
\left(\begin{array}{c}
1 \\
-i
\end{array}\right) e^{(i k z-i \omega t)}
$$

whose real part is

$$
\left(\begin{array}{l}
X \\
Y
\end{array}\right)=\left(\begin{array}{c}
\cos (k z-\omega t) \\
\sin (k z-\omega t)
\end{array}\right) .
$$

This leads to the familiar equation for the circle

$$
X^{2}+Y^{2}=1 .
$$

If the phase shifter of Eq.(99) is applied to the above Jones vector, the result is

$$
\left(\begin{array}{c}
{[\cos (\delta / 2)+\sin (\delta / 2)] \cos (k z-\omega t)} \\
i[\sin (\delta / 2)-\cos (\delta / 2)] \sin (k z-\omega t)
\end{array}\right)
$$

with

$$
\begin{aligned}
& \cos (\delta / 2)=\cos ([\delta / 2+\pi / 4]-\pi / 4), \\
& \sin (\delta / 2)=\cos ([\delta / 2+\pi / 4]+\pi / 4) .
\end{aligned}
$$

Thus, 


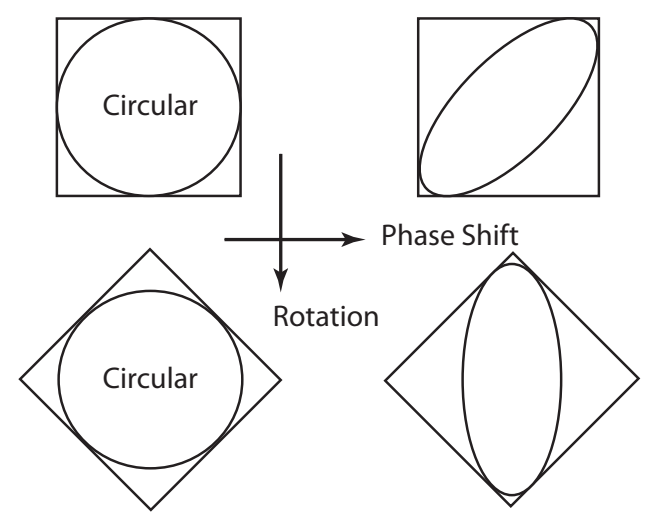

Figure 3: Phase shift and rotation. They are rotated by $45^{\circ}$.

$$
\begin{aligned}
& \cos (\delta / 2)+\sin (\delta / 2)=\sqrt{2} \cos (\delta / 2+\pi / 4), \\
& \cos (\delta / 2)-\sin (\delta / 2)=\sqrt{2} \sin (\delta / 2+\pi / 4) .
\end{aligned}
$$

After the phase shift, the Jones vector becomes

$$
\left(\begin{array}{c}
{[\sqrt{2} \cos \alpha] \cos (k z-\omega t)} \\
{[\sqrt{2} \sin \alpha] \sin (k z-\omega t)}
\end{array}\right)
$$

with

$$
\alpha=\frac{\delta}{2}+\frac{\pi}{4}
$$

The the $x$ and $y$ components will satisfy the equation

$$
\frac{X^{2}}{(\sqrt{2} \cos \alpha)^{2}}+\frac{Y^{2}}{(\sqrt{2} \sin \alpha)^{2}}=1
$$

This is an elliptic polarization.

Let us next consider rotations of the squeeze matrix

$$
B(\theta, \mu)=R(\theta) B(\mu) R(-\theta)
$$

which leads to

$$
B(\theta, \mu)=\left(\begin{array}{cc}
\cosh (\mu / 2)+\sinh (\mu / 2) \cos \theta & \sinh (\mu / 2) \sin \theta \\
\sinh (\mu / 2) \sin \theta & \cosh (\mu / 2)-\sinh (\mu / 2) \cos \theta
\end{array}\right) .
$$


From Sec. 3, we are familiar with this squeeze operation which changes the amplitudes.

If two squeeze transformations are made in two different directions, the result is not another squeeze, but a squeeze matrix followed by a rotation, which can be written as (Başkal et al. 2005)

$$
B(\theta, \lambda) B(0, \mu)=B(\phi, \xi) R(\omega),
$$

where

$$
\begin{aligned}
\cosh \xi & =\cosh \mu \cosh \lambda+\sinh \mu \sinh \lambda \cos \theta, \\
\tan \phi & =\frac{\sin \theta[\sinh \lambda+\tanh \mu(\cosh \lambda-1) \cos \theta]}{\sinh \lambda \cos \theta+\tanh \mu\left[1+(\cosh \lambda-1) \cos ^{2} \theta\right]}, \\
\tan \omega & =\frac{2(\sin \theta)\left[\sinh \lambda \sinh \mu+C_{-} \cos \theta\right]}{C_{+}+C_{-} \cos (2 \theta)+2 \sinh \lambda \sinh \mu \cos \theta},
\end{aligned}
$$

with

$$
C_{ \pm}=(\cosh \lambda \pm 1)(\cosh \mu \pm 1) .
$$

Indeed, Eq.(111) can be written as

$$
R(\omega)=B(\phi,-\xi) B(\theta, \lambda) B(0, \mu),
$$

three squeeze transformations lead to one rotation.

We have done this calculation using the kinematics of Lorentz transformations. On the other hand, it does not appear possible to do experiments using high-energy particles. However, it is gratifying to note that this experiment is possible in polarization optics.

If the angle $\theta$ is $90^{\circ}$ we use the notation $S(\lambda)$ for $B\left(\frac{\pi}{2}, \lambda\right)$. So

$$
S(\lambda)=\left(\begin{array}{cc}
\cosh (\lambda / 2) & \sinh (\lambda / 2) \\
\sinh (\lambda / 2) & \cosh (\lambda / 2)
\end{array}\right),
$$

and the above calculations become simpler with

$$
S(\lambda) B(0, \mu)=B(\phi, \xi) R(\omega),
$$


where

$$
\begin{aligned}
\cosh \xi & =\cosh \mu \cosh \lambda, \\
\tan \phi & =\frac{\sinh \lambda}{\tanh \mu}, \\
\tan \omega & =\frac{\sinh \lambda \sinh \mu}{\cosh \mu+\cosh \lambda} .
\end{aligned}
$$

\subsection{Optical Activities}

For convenience, let us change the parameters $\theta$ and $\mu$ as

$$
\theta=2 \alpha z, \quad \mu=2 \beta z,
$$

and the $R(\theta)$ and $S(\mu)$ matrices as

$$
R(\alpha z)=\left(\begin{array}{cc}
\cos (\alpha z) & -\sin (\alpha z) \\
\sin (\alpha z) & \cos (\alpha z)
\end{array}\right)
$$

and the rotation angle increased as the beam propagates along the $z$ direction. This version of optical activity is well known.

In addition, we can consider the squeeze operation

$$
S(-\beta z)=\left(\begin{array}{cc}
\cosh (\beta z) & -\sinh (\beta z) \\
-\sinh (\beta z) & \cosh (\beta z)
\end{array}\right)
$$

Here the squeeze parameter increases as the beam moves. The negative sign for $\beta$ is for convenience.

If this squeeze is followed by the rotation of Eq.(119), the net effect is

$$
\left(\begin{array}{cc}
\cosh (\beta z) & -\sinh (\beta z) \\
-\sinh (\beta z) & \cosh (\beta z)
\end{array}\right)\left(\begin{array}{cc}
\cos (\alpha z) & -\sin (\alpha z) \\
\sin (\alpha z) & \cos (\alpha z)
\end{array}\right)
$$

where $z$ is in a macroscopic scale, perhaps measured at the order of centimeters. However, this is not an accurate description of the optical process.

In fact it happens in a microscopic scale of $z / N$, and becomes accumulated into the macroscopic scale of $z$ after $N$ repetitions, where $N$ is a very large number. We are thus led to the transformation matrix of the form (Kim 2010)

$$
M(\alpha, \beta, z)=[S(-\beta z / N) R(\alpha z / N)]^{N}
$$


In the limit of large $N$, this quantity becomes

$$
\left[\left(\begin{array}{cc}
1 & -\beta z / N \\
-\beta z / N & 1
\end{array}\right)\left(\begin{array}{cc}
1 & -\alpha z / N \\
\alpha z / N & 1
\end{array}\right)\right]^{N}
$$

Since $\alpha z / N$ and $\beta z / N$ are very small,

$$
M(\alpha, \beta, z)=\left[\left(\begin{array}{ll}
1 & 0 \\
0 & 1
\end{array}\right)+\left(\begin{array}{cc}
0 & -(\alpha+\beta) \\
(\alpha-\beta) & 0
\end{array}\right) \frac{z}{N}\right]^{N} .
$$

For large $N$, we can write this matrix as

$$
M(\alpha, \beta, z)=\exp (H z)
$$

with

$$
H=\left(\begin{array}{cc}
0 & -(\alpha+\beta) \\
(\alpha-\beta) & 0
\end{array}\right) .
$$

We can compute this matrix using the procedure developed in Sec. 3. If $\alpha$ is greater than $\beta, H$ becomes

$$
H=\alpha^{\prime}\left(\begin{array}{cc}
0 & \exp (\eta) \\
\exp (-\eta) & 0
\end{array}\right)
$$

with

$$
\begin{aligned}
& \alpha^{\prime}=\sqrt{\alpha^{2}-\beta^{2}}, \\
& \exp (\eta)=\sqrt{\frac{\alpha+\beta}{\alpha-\beta}},
\end{aligned}
$$

and the $M$ matrix of Eq.(125) take the form

$$
\left(\begin{array}{cc}
\cos \left(\alpha^{\prime} z\right) & -e^{\eta} \sin \left(\alpha^{\prime} z\right) \\
e^{-\eta} \sin \left(\alpha^{\prime} z\right) & \cos \left(\alpha^{\prime} z\right)
\end{array}\right)
$$

If $\beta$ is greater than $\alpha$, the off-diagonal elements have the same sign. We can then write $H$ as

$$
H=-\beta^{\prime}\left(\begin{array}{cc}
0 & \exp (\eta) \\
\exp (-\eta) & 0
\end{array}\right)
$$


with

$$
\begin{aligned}
& \beta^{\prime}=\sqrt{\beta^{2}-\alpha^{2}}, \\
& \exp (\eta)=\sqrt{\frac{\beta+\alpha}{\beta-\alpha}},
\end{aligned}
$$

and the $M$ matrix of Eq.(125) becomes

$$
\left(\begin{array}{cc}
\cosh \left(\beta^{\prime} z\right) & -e^{\eta} \sinh \left(\beta^{\prime} z\right) \\
-e^{-\eta} \sinh \left(\beta^{\prime} z\right) & \cosh \left(\beta^{\prime} z\right)
\end{array}\right) .
$$

If $\alpha=\beta$, the lower-left element of the $H$ matrix has to vanish, and it becomes

$$
H=\left(\begin{array}{cc}
0 & -2 \alpha \\
0 & 0
\end{array}\right)
$$

and the $M$ matrix takes the triangular form

$$
\left(\begin{array}{cc}
1 & -2 \alpha z \\
0 & 1
\end{array}\right)
$$

The optical material can be made to provide rotations of the polarization axis. It is much more interesting to see this additional effect of squeeze.

\section{Stokes Parameters and the Poincaré Sphere}

In Sec. 6, we studied various aspects of the Jones vector formalism of the polarized beams, we have not dealt with the problem of whether the two beams are coherent with each other. In order to study this coherence problem we have to construct the four Stokes parameters.

Let us write the Jones vector of Eq.(80) as

$$
\left(\begin{array}{l}
\psi_{1} \\
\psi_{2}
\end{array}\right)=\left(\begin{array}{l}
a \exp \left\{i\left(k z-\omega t+\delta_{1}\right)\right\} \\
b \exp \left\{i\left(k z-\omega t+\delta_{2}\right)\right\}
\end{array}\right)
$$

where $a$ and $b$ are positive real numbers. In Sec. 6, we studied the effects of the squeeze $B(\eta)$, phase shift $Z(\delta)$, and rotation $R(\theta)$ on the Jones matrix. These matrices are given in Eq.(83), Eq. (85) and Eq.(86) respectively.

These transformation matrices can be written as one expression as the two-by-two matrix of $G$ of Eq.(65), and its role in the Lorentz group and 
its physical application to the Jones vectors were discussed in Secs. 5 and 6 , respectively. While the Jones vector can deal with two independent beams, it does not address the issue of whether they are coherent with other. For this purpose, let us introduce the coherency matrix (Brosseau 1998, Saleh et al. 2007).

$$
C=\left(\begin{array}{ll}
S_{11} & S_{12} \\
S_{21} & S_{22}
\end{array}\right)
$$

with

$$
<\psi_{i}^{*} \psi_{j}>=\frac{1}{T} \int_{0}^{T} \psi_{i}^{*}(t+\tau) \psi_{j}(t) d t
$$

where $T$ is for a sufficiently long time interval, is much larger than $\tau$. Then, those four elements become

$$
\begin{aligned}
& S_{11}=<\psi_{1}^{*} \psi_{1}>=a^{2}, \quad S_{12}=<\psi_{1}^{*} \psi_{2}>=a b e^{-(\sigma+i \delta)}, \\
& S_{21}=<\psi_{2}^{*} \psi_{1}>=a b e^{-(\sigma-i \delta)}, \quad S_{22}=<\psi_{2}^{*} \psi_{2}>=b^{2} .
\end{aligned}
$$

The diagonal elements are the absolute values of $\psi_{1}$ and $\psi_{2}$ respectively. The off-diagonal elements could be smaller than the product of $\psi_{1}$ and $\psi_{2}$, if the two beams are not completely coherent. Thus, the parameter $\sigma$ serves as the decoherence parameter.

The $\sigma$ parameter specifies the degree of coherency. Unlike the $A B C D$ matrix, this coherency matrix is not always real, and its determinant is not always one. If we restrict the trace of this matrix to be one by normalizing, this matrix becomes the density matrix (Feynman 1972).

If we start with the Jones vector of the form of Eq.(135), the coherency matrix becomes

$$
C=\left(\begin{array}{cc}
a^{2} & a b e^{-(\sigma+i \delta)} \\
a b e^{-(\sigma-i \delta)} & b^{2}
\end{array}\right)
$$

We are interested in the symmetry properties of this matrix. Since the transformation matrix applicable to the Jones vector is the two-by-two representation of the Lorentz group, we are particularly interested in the transformation matrices applicable to this coherency matrix.

The trace and the determinant of the above coherency matrix are

$$
\begin{aligned}
& \operatorname{det}(C)=(a b)^{2}\left(1-e^{-2 \sigma}\right), \\
& \operatorname{tr}(C)=a^{2}+b^{2} .
\end{aligned}
$$


Since $e^{-\sigma}$ is always smaller than one, we can introduce an angle $\chi$ defined as

$$
\cos \chi=e^{-\sigma},
$$

and call it the "decoherrence angle." If $\chi=0$, the decoherence is minimum, and it is maximum when $\chi=90^{\circ}$. We can then write the decoherency matrix of of Eq.(139) as

$$
C=\left(\begin{array}{cc}
a^{2} & a b(\cos \chi) e^{-i \delta} \\
a b(\cos \chi) e^{i \delta} & b^{2}
\end{array}\right) .
$$

The degree of polarization is defined as (Saleh et al. 2007)

$$
P=\sqrt{1-\frac{4 \operatorname{det}(C)}{(\operatorname{tr}(C))^{2}}}=\sqrt{1-\frac{\left.4(a b \sin \chi)^{2}\right)}{\left(a^{2}+b^{2}\right)^{2}}} .
$$

This degree is one if $\chi=0$. It becomes

$$
\frac{a^{2}-b^{2}}{a^{2}+b^{2}},
$$

when $\chi=90^{\circ}$. We can without loss of generality assume that $a$ is greater than $b$. If they are equal, the degree of polarization is zero.

\subsection{Stokes Parameters as Four-Vectors}

Under the influence of the $G$ transformation given in Eq.(65), this coherency matrix is transformed as

$$
\begin{aligned}
C^{\prime}= & G C G^{\dagger}=\left(\begin{array}{ll}
S_{11}^{\prime} & S_{12}^{\prime} \\
S_{21}^{\prime} & S_{22}^{\prime}
\end{array}\right) \\
& =\left(\begin{array}{ll}
\alpha & \beta \\
\gamma & \delta
\end{array}\right)\left(\begin{array}{ll}
S_{11} & S_{12} \\
S_{21} & S_{22}
\end{array}\right)\left(\begin{array}{ll}
\alpha^{*} & \gamma^{*} \\
\beta^{*} & \delta^{*}
\end{array}\right) .
\end{aligned}
$$

Here, the $G$ matrix is not Hermitian, and its Hermitian conjugate is not always its inverse. Thus it is not a similarity transformation, yet it preserves the determinant of $C$. When the $G$ matrix in Eq.(65) consists of real elements, it becomes the transformation matrix applicable to the $A B C D$ matrix. If it is constrained to be Hermitian, it becomes a rotation matrix without boosts. 
While the coherency matrix is transformed as in Eq.(145), its components transforms in the same manner as in Eq.667)

$$
\left(\begin{array}{c}
S_{11}^{\prime} \\
S_{12}^{\prime} \\
S_{21}^{\prime} \\
S_{22}^{\prime}
\end{array}\right)=\left(\begin{array}{cccc}
\alpha \alpha^{*} & \alpha \beta^{*} & \beta \alpha^{*} & \beta \beta^{*} \\
\alpha \gamma^{*} & \alpha \delta^{*} & \beta \gamma^{*} & \beta \delta^{*} \\
\gamma \alpha^{*} & \gamma \beta^{*} & \delta \alpha^{*} & \delta \beta^{*} \\
\gamma \gamma^{*} & \gamma \delta^{*} & \delta \gamma^{*} & \delta \delta^{*}
\end{array}\right)\left(\begin{array}{c}
S_{11} \\
S_{12} \\
S_{21} \\
S_{22}
\end{array}\right)
$$

Particular combinations of the coherency matrix components of Eq.(136) are crucial for the quantum picture of polarization (Falkoff et al. 1951):

$$
\begin{array}{ll}
S_{0}=\frac{S_{11}+S_{22}}{\sqrt{2}}, & S_{3}=\frac{S_{11}-S_{22}}{\sqrt{2}}, \\
S_{1}=\frac{S_{12}+S_{21}}{\sqrt{2}}, & S_{2}=\frac{S_{12}-S_{21}}{\sqrt{2} i}
\end{array}
$$

which can also be expressed as the sum of an identity matrix and the Pauli spin matrices $\sigma^{i}$ (Fano 1954)

$$
C=\frac{1}{2}\left(S_{0} I+S_{1} \sigma^{1}+S_{2} \sigma^{2}+S_{3} \sigma^{3}\right)
$$

where the coefficients are known as the Stokes parameters in the literature (Shurcliff 1962). Furthermore, expressing in such a compact form also serves to treat the Jones and Mueller calculi in the framework of pure operational Pauli algebraic approach (Tudor 2010).

The the four-by-four matrix which transforms $\left(S_{11}, S_{22}, S_{12}, S_{21}\right)$ to $\left(S_{0}, S_{3}, S_{1}, S_{2}\right)$ is

$$
\left(\begin{array}{l}
S_{0} \\
S_{3} \\
S_{1} \\
S_{2}
\end{array}\right)=\frac{1}{\sqrt{2}}\left(\begin{array}{cccc}
1 & 1 & 0 & 0 \\
1 & -1 & 0 & 0 \\
0 & 0 & 1 & 1 \\
0 & 0 & -i & i
\end{array}\right)\left(\begin{array}{c}
S_{11} \\
S_{22} \\
S_{12} \\
S_{21}
\end{array}\right)
$$

This matrix enables us to construct the transformation matrix applicable to the Stokes parameters, widely known as the Mueller matrix (Soleillet 1929, Mueller 1943, Brosseau 1998).

The Mueller matrix applicable to the Stokes parameters takes the same form as the Lorentz transformation matrix applicable to the space-time fourvector of $(t, z, x, y)$ given in Sec. 5. Therefore, the Mueller matrix is a fourby-four representation of the Lorentz group. 
It is gratifying to note that the four-by-four Mueller matrices share the same symmetry properties as those of the two-by-two Jones matrices applicable to the Jones vectors. Thanks to the squeeze matrix $B(\mu)$ of Eq. (83), we are able to extend the symmetry of those two-by-two matrices from $S U(2)$ to $S L(2, c)$ (Han et al. 1997, Devlaminck et al. 2008, Redkov 2011).

We should note here that the decoherence angle is a Lorentz-invariant quantity. It cannot be changed by Mueller transformations. It may be possible to construct a four-by-four matrix which will change this parameter (Ortega-Qujiano et al. 2011), but this matrix cannot belong to the Lorentz group.

As for the two-by-two matrix formalism, it is an iterating proposition to formulate the problem using quarternions (Dlugunovich et al. 2009, Tudor 2010). Quarternions represent a four-dimensional rotation group and do more. Thus, interesting results may be obtained from this line of approach.

\subsection{Winger's Little Group for Internal Space-time Sym- metries}

It is more interesting to study the problem using the two-by-two representation of the coherency matrix because their elements are directly measurable quantities. For this purpose, let us recall the Lorentz transformation of the four-vector of a free particle $\left(p_{0}, p_{3}, p_{1}, p_{2}\right)$ which is the same as that of the $(t, z, x, y)$ four-vector, and we can write its $G$ transformation as

$$
\begin{aligned}
P^{\prime}= & G P G^{\dagger}=\left(\begin{array}{cc}
p_{0}^{\prime}+p_{z}^{\prime} & p_{x}^{\prime}-i p_{y}^{\prime} \\
p_{x}^{\prime}+i p_{y}^{\prime} & p_{0}^{\prime}-p_{z}^{\prime}
\end{array}\right) \\
& =\left(\begin{array}{ll}
\alpha & \beta \\
\gamma & \delta
\end{array}\right)\left(\begin{array}{cc}
p_{0}+p_{z} & p_{x}-i p_{y} \\
p_{x}+i p_{y} & p_{0}-p_{z}
\end{array}\right)\left(\begin{array}{ll}
\alpha^{*} & \gamma^{*} \\
\beta^{*} & \delta^{*}
\end{array}\right) .
\end{aligned}
$$

The two-by-two matrix for the four-momentum $P$ is given in Eq.(171).

We can consider transformations which will leave the four-momentum invariant. In other words, we can write Wigner's little group as the subset of the $G$ matrix which satisfies

$$
\left(\begin{array}{cc}
p_{0}+p_{z} & p_{x}-i p_{y} \\
p_{x}+i p_{y} & p_{0}-p_{z}
\end{array}\right)=\left(\begin{array}{cc}
\alpha & \beta \\
\gamma & \delta
\end{array}\right)\left(\begin{array}{cc}
p_{0}+p_{z} & p_{x}-i p_{y} \\
p_{x}+i p_{y} & p_{0}-p_{z}
\end{array}\right)\left(\begin{array}{cc}
\alpha^{*} & \gamma^{*} \\
\beta^{*} & \delta^{*}
\end{array}\right)
$$

Using the rotation matrix $Z(\delta)$ of Eq.(85) which leads to a phase shift, we 
can bring this formula to the form

$$
\left(\begin{array}{cc}
p_{0}+p_{z} & p_{x} \\
p_{x} & p_{0}-p_{z}
\end{array}\right)=\left(\begin{array}{cc}
\alpha & \beta \\
\gamma & \delta
\end{array}\right)\left(\begin{array}{cc}
p_{0}+p_{z} & p_{x} \\
p_{x} & p_{0}-p_{z}
\end{array}\right)\left(\begin{array}{cc}
\alpha & \gamma \\
\beta & \delta
\end{array}\right)
$$

This can then be transformed to a diagonal form

$$
\left(\begin{array}{cc}
p_{0}+\sqrt{p_{z}^{2}+p_{x}^{2}} & 0 \\
0 & p_{0}-\sqrt{p_{z}^{2}+p_{x}^{2}}
\end{array}\right),
$$

with the rotation matrix $R(\xi)$, where

$$
\tan \xi=\frac{p_{x}}{p_{z}}
$$

With the boost squeeze matrix $B(\eta)$ given in Eq.(83) where

$$
e^{\eta}=\sqrt{\frac{p_{0}+p_{z}}{p_{0}-p_{z}}},
$$

we can transform the diagonal matrix of Eq.(153) to another diagonal matrix

$$
\left(\begin{array}{cc}
m & 0 \\
0 & m
\end{array}\right)
$$

where $m=\sqrt{p_{o}^{2}-p_{z}^{2}-p_{x}^{2}}$. The squeeze matrix $B(\eta)$ corresponds the Lorentz boost given in Eq.(72).

The $G$ transformation matrix which will leave this four-momentum matrix is has to be Hermitian, and is a rotation matrix $R(\theta)$. This defines Wigner's internal space-time symmetry of a massive particle.

This matrix remains invariant under the $G$ transformation if the $G$ matrix is Hermitian. It is Hermitian only for rotations. This is thus consistent with Wigner's $O(3)$-like little group for massive particles, as discussed in Sec. 5 ,

For a massless particle, we can choose the system where $p_{0}=p_{z}=\omega$ and $p_{x}=p_{y}=0$. Then the $P$ matrix becomes

$$
P=\left(\begin{array}{cc}
2 \omega & 0 \\
0 & 0
\end{array}\right)
$$

and its determinant is zero, saying $p_{0}^{2}-p_{z}^{2}=0$. It is not difficult to construct the $G$ matrix whose $G$ transformation will leave the above $P$ matrix invariant. It takes triangular form

$$
\left(\begin{array}{ll}
1 & \gamma \\
0 & 1
\end{array}\right)
$$


From this, it is not difficult to construct its four-by-four counterpart given in Eq.(178).

Let us go back to the symmetry of the coherency matrix, and to the matrix $C$ of Eq.(139). If we make a $G$ transformation with $Z(\delta)$, the $D$ matrix becomes

$$
\left(\begin{array}{cc}
a^{2} & a b \cos \chi \\
a b \cos \chi & b^{2}
\end{array}\right),
$$

The two-by-two matrix of $Z(\delta)$ is given in Eq. (85).

If we make another $G$ transformation with $R(-\theta)$ where

$$
\tan \theta=\frac{2 a b \cos \chi}{a^{2}-b^{2}},
$$

the coherency matrix becomes

$$
C=\left(\begin{array}{cc}
s+r & 0 \\
0 & s-r
\end{array}\right),
$$

with

$$
\begin{aligned}
& s=\frac{1}{2}\left(a^{2}+b^{2}\right), \\
& r=\frac{1}{2} \sqrt{\left(a^{2}+b^{2}\right)^{2}+4(a b)^{2} \sin ^{2} \chi} .
\end{aligned}
$$

If $\theta=0$, the system is totally coherent, and the coherency matrix becomes

$$
\left(\begin{array}{cc}
a^{2}+b^{2} & 0 \\
0 & 0
\end{array}\right),
$$

This matrix is like the four-momentum matrix of Eq.(71) for massless particles.

If $\chi$ is nonzero, we can $G$-transform the $D$ matrix of with

$$
B(-\eta)=\left(\begin{array}{cc}
e^{-\eta / 2} & 0 \\
0 & e^{\eta / 2}
\end{array}\right),
$$

with

$$
e^{\eta}=\sqrt{\frac{s+r}{s-r}},
$$

the $D$ matrix becomes

$$
C=\left(\begin{array}{cc}
\sqrt{s^{2}-r^{2}} & 0 \\
0 & \sqrt{s^{2}-r^{2}}
\end{array}\right),
$$


This coherency matrix is invariant under $G$ transformations if the $G$ matrix consists only of rotations and thus is Hermitian. This aspect is consistent with Wigner's $O(3)$-like little group for massive particles.

In the case of the four-momentum matrix, its determinant is $m^{2}$ and is Lorentz-invariant. For the coherency matrix, the determinant is $(a b)^{2} \sin ^{2} \chi$. This means that the coherency angle $\chi$ cannot be changed by Lorentz transformations, as in the case of mass in special relativity.

\section{Symmetries of the Poincaré Sphere}

The Poincaré sphere has a long history, and its spherical symmetry is well known (Born et al. 1980). The rotational symmetry of the Poincaré sphere is readily included in the Lorentz symmetry. We shall first review the rotational symmetry, and study the effect of Lorentz boosts.

Let us write the coherency matrix of Eq.(139) as a four-component vector

$$
\left(\begin{array}{c}
s \\
r_{z} \\
r_{x} \\
r_{y}
\end{array}\right)=\left(\begin{array}{c}
s \\
r(\cos \theta) \\
r(\sin \theta) \cos \delta \\
r(\sin \theta) \sin \delta
\end{array}\right)=\left(\begin{array}{c}
\left(a^{2}+b^{2}\right) / 2 \\
\left(a^{2}-b^{2}\right) / 2 \\
a b(\cos \delta) \cos \chi \\
a b(\sin \delta) \cos \chi
\end{array}\right) .
$$

This four-vector is defined by two spheres: the outer sphere with radius $s$, where

$$
s=\frac{\left(a^{2}+b^{2}\right)}{2}
$$

and the inner sphere defined by the three-component vector $\left(r_{z}, r_{x}, r_{y}\right)$, with its radius

$$
\begin{aligned}
r & =\sqrt{r_{z}^{2}+r_{x}^{2}+r_{y}^{2}} \\
& =\frac{1}{2} \sqrt{\left(a^{2}-b^{2}\right)^{2}+4(a b)^{2} \cos ^{2} \chi}
\end{aligned}
$$

which is the radius of the Poincaré sphere. Its $z$ component is

$$
r_{z}=\frac{a^{2}-b^{2}}{2}
$$

which is independent of the decoherency angle $\chi$. Here we assume the amplitude $a$ to be greater than $b$. 
The radius of the Poincaré sphere $r$ depends on $\chi$, and takes its maximum value $s$ when $\chi=0$. The radius shrinks to its minimum value $r_{z}$ when $\chi$ goes to it largest value. Figure 4 illustrates the circles and their radii. The ratio of $r_{z}$ to $r$ is

$$
\cos \theta=\frac{r_{z}}{r}=\frac{a^{2}-b^{2}}{\sqrt{\left(a^{2}-b^{2}\right)^{2}+4(a b)^{2} \cos ^{2} \chi}} .
$$

If we apply the rotation

$$
\left(\begin{array}{cccc}
1 & 0 & 0 & 0 \\
0 & 1 & 0 & 0 \\
0 & 0 & \cos \delta & \sin \delta \\
0 & 0 & -\sin \delta & \cos \delta
\end{array}\right)
$$

and then

$$
\left(\begin{array}{cccc}
1 & 0 & 0 & 0 \\
0 & \cos \theta & \sin \theta & 0 \\
0 & -\sin \theta & \cos \theta & 0 \\
0 & 0 & 0 & 1
\end{array}\right)
$$

with $\cos \theta=r_{z} / r$ given in Eq.(170), this four-vector can be brought into the form

$$
(s, r, 0,0) .
$$

Within the framework of the traditional three-dimensional geometry of the Poincaré sphere, it is possible to transform the four vector of Eq.(166) to the four vector of Eq.(173) while the value of $s$ is left unchanged. On the other hand, the Lorentz symmetry allows a transformation on this fourvector by $B(-\eta)$ with $\tanh \eta=r / s$ so that the four-vector becomes (Başkal et al. 2006)

$$
\left(\begin{array}{c}
\sqrt{s^{2}-r^{2}} \\
0 \\
0 \\
0
\end{array}\right)=\left(\begin{array}{cccc}
\cosh \eta & -\sinh \eta & 0 & 0 \\
-\sinh \eta & \cosh \eta & 0 & 0 \\
0 & 0 & 1 & 0 \\
0 & 0 & 0 & 1
\end{array}\right)\left(\begin{array}{l}
s \\
r \\
0 \\
0
\end{array}\right)
$$

This means that the radius of the Poincaré sphere can become zero while the outer radius takes its minimum value $a b \sin \chi$.

Indeed, the angle $\chi$ determines the minimum radius of the outer sphere. This radius takes the maximum value of $a b$ when $\sigma$ becomes infinity. The 
larger radius becomes zero when $\sigma=0$ corresponding to completely coherent beams, and it does not correspond to the real world.

In order to resolve this problem, we note that the large radius and smaller radius are the same and its value is $\left(a^{2}+b^{2}\right) / 2$, when $\chi=0$. Thus, if we make the Lorentz boost of Eq.(174), the result is

$$
e^{-\eta}\left(\begin{array}{c}
s \\
s \\
0 \\
0
\end{array}\right)=\left(\begin{array}{cccc}
\cosh \eta & -\sinh \eta & 0 & 0 \\
-\sinh \eta & \cosh \eta & 0 & 0 \\
0 & 0 & 1 & 0 \\
0 & 0 & 0 & 1
\end{array}\right)\left(\begin{array}{l}
s \\
s \\
0 \\
0
\end{array}\right)
$$

The Lorentz group, including its rotation subgroup, changes all the parameters for the coherency matrix. However, it cannot change the decoherence angle $\chi$. What significance does this carry from the symmetry point of view and from the physical point of view?

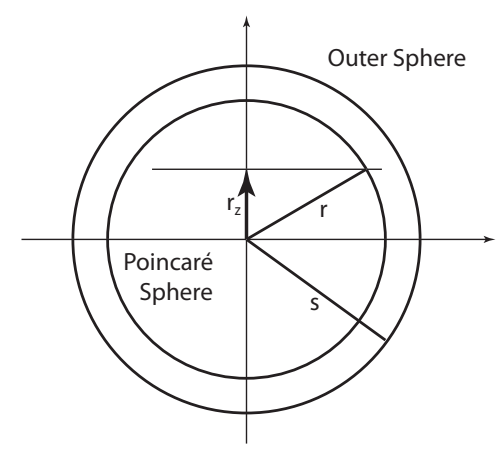

Figure 4: Poincaré sphere and its outer sphere. The radius of the Poincaré sphere depends on the decoherence angle $\chi$, but its z component does not.

\section{1 $\mathrm{O}(3,2)$ Symmetry of the Poincaré Sphere}

It is clear from the previous subsection that the decoherence angle $\chi$ is invariant under Lorentz transformations. We are now looking for another symmetry which will change this variable. For this purpose we write the coherency matrix of Eq.(142) as

$$
C_{1}=\left(\begin{array}{cc}
a^{2} & a b(\cos \chi) e^{-i \delta} \\
a b(\cos \chi) e^{i \delta} & b^{2}
\end{array}\right)
$$


and introduce another matrix where $\cos \chi$ is replaced by $\sin \chi$, which can take the form

$$
C_{2}=\left(\begin{array}{cc}
a^{2} & a b(\sin \chi) e^{-i \delta} \\
a b(\sin \chi) e^{i \delta} & b^{2}
\end{array}\right) .
$$

For the first coherency matrix of Eq.(176), we know how to transform it into the four-vector $\left(s, r_{1}, 0,0\right)$, with

$$
s=\frac{a^{2}+b^{2}}{2}, \quad r_{1}=\frac{1}{2} \sqrt{\left(a^{2}-b^{2}\right)^{2}-4(a b)^{2}(\cos \chi)^{2}} .
$$

Then, the second matrix can be brought to the four-vector $\left(u, r_{2}, 0,0\right)$, with

$$
u=\frac{a^{2}+b^{2}}{2}, \quad r_{2}=\frac{1}{2} \sqrt{\left(a^{2}-b^{2}\right)^{2}-4(a b)^{2}(\sin \chi)^{2}} .
$$

These two expressions lead us to consider the following two four vectors.

$$
\left(\begin{array}{c}
a b(\cos \chi) \\
0 \\
0 \\
0
\end{array}\right), \quad\left(\begin{array}{c}
a b(\sin \chi) \\
0 \\
0 \\
0
\end{array}\right) .
$$

In view of the relation $\cos ^{2} \chi+\sin ^{2} \chi=1$, we are led to the five dimensional vector space with $\left(s, u, r_{z}, r_{x}, r_{y}\right)$, which can start with

$$
(a b \cos \chi, a b \sin \chi, 0,0,0) .
$$

Now we can change the value of the decoherence parameter $\sigma$ by changing the angle $\chi$, but we can change this variable by introducing a rotation matrix applicable to the two-dimensional vector space of $s$ and $t$. When all other components vanish we can write the rotation matrix

$$
\left(\begin{array}{c}
a b(\cos \chi) \\
a b(\sin \chi)
\end{array}\right)=\left(\begin{array}{cc}
\cos \chi & -\sin \chi \\
\sin \chi & \cos \chi
\end{array}\right)\left(\begin{array}{c}
a b \\
0
\end{array}\right) .
$$

Therefore the five-by-five rotation matrix will be of the form

$$
\left(\begin{array}{ccccc}
\cos \chi & -\sin \chi & 0 & 0 & 0 \\
\sin \chi & \cos \chi & 0 & 0 & 0 \\
0 & 0 & 1 & 0 & 0 \\
0 & 0 & 0 & 1 & 0 \\
0 & 0 & 0 & 0 & 1
\end{array}\right)
$$


which is applicable to the five-component vector $\left(s, t, r_{z}, r_{x}, r_{y}\right)$, with the two four-dimensional subspaces, corresponding to the coherency matrices

$$
\begin{aligned}
& C_{s}(\chi)=\left(\begin{array}{cc}
a^{2} & a b e^{-i \delta}(\cos \chi) \\
b a e^{i \delta}(\cos \chi) & b^{2}
\end{array}\right), \\
& C_{t}(\chi)=\left(\begin{array}{cc}
a^{2} & a b e^{-i \delta}(\sin \chi) \\
b a e^{i \delta}(\sin \chi) & b^{2}
\end{array}\right) .
\end{aligned}
$$

Here both $s^{2}-r^{2}$ and $t^{2}-r^{2}$ remain invariant under Lorentz transformations within their four-dimensional subspaces. Their deteminants are

$$
\operatorname{det}\left(C_{s}\right)=(a b)^{2} \sin ^{2} \chi, \quad \operatorname{det}\left(C_{u}\right)=(a b)^{2} \cos ^{2} \chi
$$

resuting in

$$
\operatorname{det}\left(C_{s}\right)+\operatorname{det}\left(C_{s}\right)=(a b)^{2},
$$

which is independent of the angle $\chi$.

\subsection{Entropy Probem}

Another important way to measure the lack of coherence is to calculate the entropy of the system. The coherency matrices defined in this section become density matrices if their traces are normalized to be one. Thus from Eq.(177), we can write

$$
\begin{aligned}
& \rho_{s}(\chi)=\frac{1}{a^{2}+b^{2}}\left(\begin{array}{cc}
a^{2} & a b e^{-i \delta}(\cos \chi) \\
b a e^{i \delta}(\cos \chi) & b^{2}
\end{array}\right), \\
& \rho_{u}(\chi)=\frac{1}{a^{2}+b^{2}}\left(\begin{array}{cc}
a^{2} & a b e^{-i \delta}(\sin \chi) \\
b a e^{i \delta}(\sin \chi) & b^{2}
\end{array}\right) .
\end{aligned}
$$

These matrices can be diagonalized to

$$
\begin{aligned}
& \rho_{s}(\chi)=\frac{1}{2}\left(\begin{array}{cc}
1+f_{s} & 0 \\
0 & 1-f_{s}
\end{array}\right), \\
& \rho_{u}(\chi)=\frac{1}{2}\left(\begin{array}{cc}
1+f_{u} & 0 \\
0 & 1-f_{u}
\end{array}\right),
\end{aligned}
$$

where

$$
f_{s}=\sqrt{1-\frac{4(a b)^{2} \sin ^{2} \chi}{a^{2}+b^{2}}}, \quad f_{u}=\sqrt{1-\frac{4(a b)^{2} \cos ^{2} \chi}{a^{2}+b^{2}}}
$$


Then, their entropies become

$$
\begin{aligned}
& S_{s}=-\left(\frac{1+f_{s}}{2}\right) \ln \left(\frac{1+f_{s}}{2}\right)-\left(\frac{1-f_{s}}{2}\right) \ln \left(\frac{1-f_{s}}{2}\right), \\
& S_{u}=-\left(\frac{1+f_{u}}{2}\right) \ln \left(\frac{1+f_{u}}{2}\right)-\left(\frac{1-f_{u}}{2}\right) \ln \left(\frac{1-f_{u}}{2}\right) .
\end{aligned}
$$

The entropy $S_{s}$ becomes zero when $\chi=0$. It becomes

$$
\frac{a^{2}}{a^{2}+b^{2}} \ln \left(\frac{a^{2}+b^{2}}{a^{2}}\right)+\frac{b^{2}}{a^{2}+b^{2}} \ln \left(\frac{a^{2}+b^{2}}{b^{2}}\right),
$$

when $\chi=90^{\circ}$. The entropy $S_{s}$ is a monotonically increasing function of the angle $\chi$ starting from zero to the above maximum value, which becomes $\ln 2$ when $a=b$.

The entropy $S_{s}$ of the first space is monotonically increasing function of $\chi$, while that of the second space $S_{u}$ is a decreasing function. Thus, an increase in entropy in the first space leads to a decrease in the second space. Then we can ask whether the sum of these two entropies becomes independent of $\chi$, leading to an entropy conservation of the total system. The answer is No. However, this does not cause problems for us, because the second space is not necessarily a physical space. It could be meaningless to use the same definition of entropy for this space. On the other hand, as we noted before, we can define the conservation of entropy in terms of the the sum of the determinants of the coherency matrices given in Eq.(184). Furthermore, this determinant condition does not require that the amplitudes of the two beams to be the same.

\subsection{Feynman's Rest of the Universe}

What is the meaning of this second space? In his book on statistical mechanics, Feynman makes the following statement about the density matrix (Feynman 1972): When we solve a quantum-mechanical problem, what we really do is divide the universe into two parts - the system in which we are interested and the rest of the universe. We then usually act as if the system in which we are interested comprised the entire universe. To motivate the use of density matrices, let us see what happens when we include the part of the universe outside the system. 
Feynman did not specify whether the rest of the universe is observable or not. In either case, it is an interesting exercise to construct a model of the rest of the universe behaving like a physical world. With this point in mind, one of us studied two coupled harmonic oscillators in which one of the oscillators correspond to the physical world and the other to the rest of the universe (Han et al. 1999). In this example, the rest of the universe is the same as the world in which we do physics. In thermal field theory (Umezawa et al. 1982), even though based on the same mathematics as that of the coupled oscillators, the rest of the universe is not physically identified, except that it causes thermal excitations of the oscillators in the physical world.

The concept of decoherence occupies one of the central places in the current development of physics. In (Feynman et al. 1963) when the system couples to finite temperature baths the result is an environmentally induced decoherence. Its effects can be determined in tunneling processes (Caldeira et al. 1983) and in two-state systems that are coupled to dissipative environments (Leggett et al. 1987). The decoherence of the electromagnetic field coupling resonantly to a two-level system (Anastopoulos et al. 2000) and applications of two-level decoherence to qubit systems (Shiokawa et al. 2004) are also investigated in the literature.

The pattern for the two-optical beams arising from phase-randomizing process (McAlister et al. 1997), is in the same structure of the two-by-two matrix discussed in this paper. As for the decoherence in the rest of the universe introduced in this work, the system becomes more coherent as the time-variable increases. Although this "recoherence" process was considered earlier in the literature (Anglin et al. 1996), it is premature to expect a two-state system to gain coherence in the real world. It is thus very safe to say that the second Minkowskian space introduced in this paper remains in Feynman's rest of the universe.

However, this does not prevent us from constructing a physical system analogous to the decoherent system coupled to a recoherent system.

\section{Concluding Remarks}

In this paper, we have organized ray and polarization optics using the language of the Lorentz group. The Lorentz group has two-by-two and four-byfour representations. Both are useful in optics, and they allow us to gain a unified view of various aspects of optics. 
In addition, it was noted that the mathematics applicable to ray and polarization optics is directly applicable to the internal space-time symmetries of elementary particles. Optical systems are favorable in the sense that each mathematical operation has a counterpart that can be performed in optics laboratories.

The Lorentz group is also the basic mathematical language for Einstein's special relativity. Currently, this group serves useful purposes in many other branches of physics, including optical sciences. In recent years, the Lorentz group served as the underlying language for squeezed states of light. It was Dirac who first observed that the Lorentz boost is a squeeze transformation (Dirac 1949) and constructed representations of the Lorentz group using coupled harmonic oscillators (Dirac 1963). Indeed, Dirac's oscillator representation forms the theoretical foundations of squeezed states of light (Yuen 1976, Yurke et al. 1986, Kim et al. 1991). This aspect of the Lorentz group is by now well known in the optics community, and the Lorentz group is one of the theoretical tools in quantum optics.

The squeezed state is not the only branch of optics requiring the Lorentz group. It can well be applied to Fourier optics (Bacry et al. 1981) while its geometry has proven to be useful in designing three dimensional non-imaging concentrators (Gutierrez et al. 1996). Para-axial wave optics (Sudarshan et al.1983, Makunda et al. 1983) and Wavelets (Han et al. 1995) are also known to be representations of this group. It is also the underlying language for reflections and refractions (Pellat-Finet et al. 1992).

\section{References}

Anastopoulos, C. and Hu, B. L. 2000. Two-level atom-field interaction: Exact master equations for non-Markovian dynamics, decoherence, and relaxation. Phys. Rev. A 62: 33821-13.

Anglin, J. R. and Zurek, W. H. 1996. Decoherence of quantum fields: Pointer states and predictability. Phys. Rev. D 53: 7327-35.

Azzam, R. A. M. and Bashara, I. 1997. Ellipsometry and Polarized Light. Amsterdam: North-Holland.

Bacry, H. and Cadilhac, M. 1981. Metaplectic group and Fourier optics. Phys. Rev. A 23: 2533-36. 
Bargmann, V. 1947. Irreducible representations of the Lorentz group. Ann. Math. 48: 568-640.

Başkal, S. and Kim, Y. S. 2005. Rotations associated with Lorentz boosts. J.Phys. A 38: 6545-56.

Başkal, S. and Kim, Y. S. 2006. de Sitter group as a symmetry for optical decoherence. J. Phys. A 39: 7775-88.

Başkal, S. and Kim, Y. S. 2009. ABCD matrices as similarity transformations of Wigner matrices and periodic systems in optics. J. Opt. Soc. Am. A 26: 3049-54.

Başkal, S. and Kim, Y. S. 2010 One analytic form for four branches of the ABCD matrix. J. Mod. Opt. 57: 1251-59.

Ben-Aryeh, Y. 2005. Nonunitary squeezing and biorthogonal scalar products in polarization optics. J. Opt. B: Quantum Semiclass. 7: S45257 .

Born, M. and Wolf, E. 1980. Principles of Optics. 6th Ed. Pergamon, Oxford.

Brosseau, C. 1998. Fundamentals of Polarized Light: A Statistical Optics Approach. New York: John Wiley.

Caldeira, A. O. and Leggett, A. J. 1983. Quantum tunneling in a dissipative system. Ann. Phys. (N.Y.) 149: 374-456.

Devlaminck, V. and P. Terrier, P. 2008. Definition of a parametric form of nonsingular Mueller matrices. J. Opt. Soc. Am. A 25: 2636-43.

Dirac, P. A. M. 1949. Forms of relativistic dynamics. Rev. Mod. Phys. 21: 392-99.

Dirac, P. A. M. 1963. A Remarkable representation of $3+2$ de Sitter group. J. Math. Phys. 4: 901-09.

Falkoff, D. L. and MacDonald J. E. 1951. On the Stokes parameters for polarized radiation. J. Opt. Soc. Am. 41: 861-62.

Dlugunovich, V. A. and Kurochkin, Y. A. 2009. Vector parameterization of the Lorentz group transformations and polar decomposition of Mueller matrices. Optics and Spectroscopy 107: 312-17.

Fano, U. 1954. A Stokes-Parameter Technique for the treatment of po- 
larization in quantum mechanics. Phys. Rev. 93: 121-23.

Feynman, R. P. and Vernon, F. L. 1963. The theory of a general quantum system interacting with a linear dissipative system. Ann. Phys. (N.Y.) 24: 118-173.

Feynman, R. P. 1972. Statistical Mechanics. Reading, MA: Benjamin/Cummings.

Georgieva, E. and Kim, Y. S. 2001. Iwasawa effects in multilayer optics. Phys. Rev. E 64: 26602-06.

Georgieva, E. and Kim, Y. S. 2003. Slide-rule-like property of Wigner's little groups and cyclic $S$ matrices for multilayer optics. Phys. Rev. E 68: 026606-12.

Gutierrez, M., Minano, J.C., Vega, C. and Benitez, P. 1996. Application of Lorentz geometry to non-imaging optics: New three-dimensional ideal contractors. J. Opt. Soc. Am. A 13: 532-42.

Han, D., Kim, Y. S. and D. Son, D. 1986. Eulerian parametrization of Wigner little groups and gauge transformations in terms of rotations in 2-component spinors. J. Math. Phys. 27: 2228-35.

Han, D., Kim, Y. S. and Noz, M. E. 1995. Wavelets, windows, and photons. Phys. Lett. A 206: 299-304.

Han, D., Kim, Y. S. and Noz, M. E. 1997. Stokes parameters as a Minkowskian four-vector. Phys. Rev. E 56: 6065-76.

Han, D., Kim, Y. S. and Noz, M. E. 1999. Illustrative example of Feynman's rest of the universe Am. J. Phys. 67: 61-66.

Haus, H. A. 1984. Waves and Fields in Optoelectronics. Englewood Cliffs, New Jersey: Prentice-Hall.

Hawkes, J. and Latimer, I. 1995 Lasers: Theory and Practice. New York: Prentice-Hall.

Hecht, E. 1970. Note on an operational definition of Stokes parameters. Am. J. Phys. 38: 1156-58.

Jones, R. C. 1941. New calculus for the treatment of optical systems. J. Opt. Soc. Am. 31: 488-93.

Jones, R. C. 1947. A new calculus for the treatment of optical systems. 
5. A more general formulation, and description of another calculus. J. Opt. Soc. Am. 37: 107-10.

Kim, Y. S. and Noz, M. E. 1986. Theory and Applications of the Poincaré Group. Dordrecht: Reidel.

Kim, Y. S. and Noz, M. E. 1991. Phase Space Picture of Quantum Mechanics. Singapore: World Scientific.

Kim, Y. S. and Wigner, E. P. 1990. Space-time geometry of relativisticparticles. J. Math. Phys. 31: 55-60.

Kim, Y. S. 2010. Optical activities as computing resources for spacetime symmetries. J. Mod. Op. 7: 17-22.

Leggett, A. J., Chakravarty, S., Dorsey, A. T., Fisher, M. P. A., Garg, A. and Zwerger, W. 1987. Dynamics of the dissipative 2-state system. Rev. Mod. Phys. 59: 1-85.

Makunda, N., Simon, R. and Sudarshan, E. C. G. 1983. Paraxial-wave optics and relativistic front description. II. The vector theory. Phys. Rev. A 28: 2933-42.

McAlister, D. F. and Raymer, M. G. 1997. Ultrafast photon-number correlations from dual-pulse, phase-averaged homodyne detection. Phys. Rev. A. 55: R1607-12.

Monzón, J. J. and Sánchez-Soto, L. L. 2000. Fresnel formulas as Lorentz transformations. J. Opt. Soc. Am. A 17: 1475-81.

Monzón, J. J., Yonte, T., Sánchez-Soto, L. L. and Carinena, J. F. 2002. Geometrical setting for the classification of multilayers. J. Opt. Soc. Am. A 19: 985-91.

Mueller, H. 1943. Memorandum on the polarization optics of the photo elastic shutter. Reprt no. 2 of OSRD, prjectOEMsr-576, November 15.

Opatrny, T. and Perina, J. 1993. Non-image-forming polarization optical devices and Lorentz transformations an analogy. Phys. Lett. A 181: 199-202.

Ortega-Quijiano, N. and Arce-Diego, J. L. 2011. Depolarizing differential Mueller matrices. Opt. Lett. 36: 2429-31.

Pellat-Finet, P. and M. Buasset, M. 1992. What is common to both 
polarization optics and relativistic kinematics? Optik 90: 101-06.

Poincarè, H. 1982. Théorie Mathématique de la Lumiere. Vol: 2. Paris: Gauthiers-Villars.

Redkov, V. M. 2011. Lorentz group theory and polarization of light. Adv. Appl. Clifford Algebras 21: 203-20.

Saleh, B. E. A. and Teich, M. C. 2007. Fundamentals of Photonics. 2nd Ed. Hoboken, New Jersey: John Wiley.

Shiokawa, K. and $\mathrm{Hu}$, B. L. 2004. Qubit decoherence and nonMarkovian dynamics at low temperatures via an effective spin-boson model. Phys. Rev. A. 70: 062106-10.

Shurcliff, W. A. 1962. Polarized Light. Cambridge, MA: Harvard Univ. Press.

Soleillet, P. 1929. Sur les paramtres caractrisant la polarisation partielle de la lumire dans les phnomnes de fluorescence. Ann. Phys. 12: 23-97.

Stokes, G. G. 1852. On the composition and resolution of streams of polarized light from different sources. Trans. Camb. Phil. Soc. 9: 399416.

Sudarshan, E. C. G., Simon, R. and Makunda, N. 1983. Paraxial-wave optics and relativistic front description. I. The scalar theory. Phys. Rev. A 28: 2921-32.

Tudor, T. 2010. Vectorial Pauli algebraic approach in polarization optics. I. Device and state operators. Optik 121: 1226-35.

Umezawa, H., Matsumoto, H. and Tachiki, M. 1982. Thermo Field Dynamics and Condenced States. Amsredam: North-Holland.

Wigner, E. 1939. On unitary representations of the inhomogeneous Lorentz group. Ann. Math. 40: 149-204.

Yariv, A. 1975. Quantum Electronics. New York: Wiley.

Yuen, H. P. 1976. Two-photon coherent states of the radiation fields. Phys. Rev. A 13: 2226-43.

Yurke, B., McCall, S. L. and Klauder, J. R. 1986. $S U(2)$ and $S U(1,1)$ interferometers. Phys. Rev. A 33: 4033-54. 